\title{
Transmission of amyloid-beta and tau pathologies is associated with cognitive impairments in a primate
}

\author{
Suzanne Lam, ${ }^{1,2}$, Fanny Petit ${ }^{1,2}$, Anne-Sophie Hérard ${ }^{1,2}$, Susana Boluda ${ }^{3,4}$, Sabiha Eddarkaoui ${ }^{5}$, \\ Martine Guillermier ${ }^{1,2}$, The Brain Bank Neuro-C. E. B. Neuropathology Network, Luc Buée ${ }^{5}$, \\ Charles Duyckaerts ${ }^{3,4}$, Stéphane Haïk ${ }^{3,4}$, Jean-Luc Picq ${ }^{1,2,6}$ and Marc Dhenain ${ }^{1,2^{*}}$ (1)
}

\begin{abstract}
Amyloid- $\beta$ (AB) pathology transmission has been described in patients following iatrogenic exposure to compounds contaminated with $A \beta$ proteins. It can induce cerebral $A \beta$ angiopathy resulting in brain hemorrhages and devastating clinical impacts. latrogenic transmission of tau pathology is also suspected but not experimentally proven. In both scenarios, lesions were detected several decades after the putatively triggering medico-surgical act. There is however little information regarding the cognitive repercussions in individuals who do not develop cerebral hemorrhages. In the current study, we inoculated the posterior cingulate cortex and underlying corpus callosum of young adult primates (Microcebus murinus) with either Alzheimer's disease or control brain extracts. This led to widespread A $\beta$ and tau pathologies in all of the Alzheimer-inoculated animals following a 21-month-long incubation period $(n=12)$ whereas none of the control brain extract-inoculated animals developed such lesions $(n=6)$. A $\beta$ deposition affected almost all cortical regions. Tau pathology was also detected in A $\beta$-deposit-free regions distant from the inoculation sites (e.g. in the entorhinal cortex), while some regions adjacent, but not connected, to the inoculation sites were spared (e.g. the occipital cortex). Alzheimer-inoculated animals developed cognitive deficits and cerebral atrophy compared to controls. These pathologies were induced using two different batches of Alzheimer brain extracts. This is the first experimental demonstration that tau can be transmitted by human brain extracts inoculations in a primate. We also showed for the first time that the transmission of widespread $A \beta$ and tau pathologies can be associated with cognitive decline. Our results thus reinforce the need to organize a systematic monitoring of individuals who underwent procedures associated with a risk of $A \beta$ and tau iatrogenic transmission. They also provide support for Alzheimer brain-inoculated primates as relevant models of Alzheimer pathology.
\end{abstract}

Keywords: Amyloid- $\beta$ pathology, Alzheimer's disease, Cerebral atrophy, Prion, Tau pathology, Transmission

\section{Introduction}

Prion diseases can occur after iatrogenic transmission of misfolded prion proteins. The aberrant proteins propagate by imposing their abnormal conformation on the

\footnotetext{
*Correspondence: marc.dhenain@cea.fr

${ }^{1}$ Laboratoire des Maladies Neurodégénératives, Université Paris-Saclay, CEA, CNRS, 18 Route du Panorama, 92265 Fontenay-aux-Roses, France

Full list of author information is available at the end of the article
}

homologous normal host cell proteins which are continually produced in the natural course of cellular metabolism. Neuropathological observational studies in humans have suggested that amyloid- $\beta(A \beta)$ pathology is transmissible through a similar mechanism to that of acquired prion diseases [17]. To date, 76 cases of $A \beta$ pathology have been reported following exposure to cadaversourced human growth hormones [9, 17], dura mater grafts [14] or after cerebral surgeries with instruments original author(s) and the source, provide a link to the Creative Commons licence, and indicate if changes were made. The images or other third party material in this article are included in the article's Creative Commons licence, unless indicated otherwise in a credit line to the material. If material is not included in the article's Creative Commons licence and your intended use is not permitted by statutory regulation or exceeds the permitted use, you will need to obtain permission directly from the copyright holder. To view a copy of this licence, visit http://creativecommons.org/licenses/by/4.0/. The Creative Commons Public Domain Dedication waiver (http://creativeco mmons.org/publicdomain/zero/1.0/) applies to the data made available in this article, unless otherwise stated in a credit line to the data. 
contaminated with $A \beta[9,21]$. The pathology occurred as $A \beta$ plaques in the brain as well as vascular $A \beta$ pathology that could be associated with fatal cerebral hemorrhages. Observational evidence of tau iatrogenic transmission is not as widely reported as for $A \beta$. A recent article detected tau lesions after incubation periods exceeding three decades in patients with iatrogenic $A \beta$ pathology [16]. However, because of the long incubation time in humans, it remains difficult to determine whether the $\mathrm{A} \beta$ and tau pathologies were really transmitted. Another critical question is whether, in the absence of severe cerebral hemorrhages, $A \beta$ and/or tau transmissions can lead to cognitive impairments.

Experimental studies in transgenic mice overexpressing $\mathrm{A} \beta$ precursor protein (APP) have shown transmissibility of $A \beta$ pathology after the intracerebral inoculation of Alzheimer's disease (AD) brain extracts [26]. Transmission of tau pathology is also described in mice overexpressing mutated tau proteins [6]. The host in which the proteopathic seeds are inoculated provides the biochemical and physiological environment that modulates lesion emergence and functional impact [18]. Transgenic mouse models of $A \beta$ or tau pathology rely on high $A \beta$ production or mutated tau protein expression, respectively. Thus, one key limitation of these models is that they provide a very different brain environment from the one found in human brains. Because of their phylogenetic proximity, primates have a brain environment closer to the human brain. A long-term study on marmosets (Callithrix jacchus) revealed the induction of sparse amyloidosis 3.5 years after intracerebral inoculations of $\mathrm{AD}$ brain homogenates [2, 23, 34]. However, tau pathologies or other AD-like features were not reported, even seven years later.

Mouse lemurs (Microcebus murinus) are small primates with an $A \beta_{1-42}$ sequence that is homologous to that of humans [37], while mice differ by three amino acids [8]. Protein sequence issued from gene for Microtubule Associated Protein Tau (MAPT) has 94.3\% identity with human gene, while murine MAPT gene has $88.8 \%$ identity (Additional File 1: Fig. S1a). Recently, our group showed that following an 18-month-incubation period, AD brain inoculations in the hippocampus and overlying cortex of mouse lemurs can lead to cognitive decline, functional alterations and cerebral atrophy associated with neuronal loss, but very sparse $A \beta$ and tau deposits [11]. In the present study, we inoculated the posterior cingulate cortex and underlying corpus callosum of young adult mouse lemurs with either AD or control brain extracts. The posterior cingulate cortex was chosen as it is a functional cerebral hub in mouse lemurs [10]. The corpus callosum was chosen based on the assumption that migration of seeds could follow white matter tracts. As fragmentation of $\mathrm{A} \beta$ seeds by extended sonication was shown to increase seeding capacity of brain extracts [20], we carefully sonicated the inoculated samples. Following a 21-month incubation period, all of ADinoculated mouse lemurs $(n=12)$ developed extensive $A \beta$ and tau pathologies in several brain regions, providing evidence for the spreading of these pathologies in the brain. Animals inoculated with control-brain extracts did not develop any A $\beta$ plaques or tau deposits. Similar results were replicated using two different types of $\mathrm{AD}$ brain extracts. AD-inoculated animals also developed progressive cognitive impairments and cerebral atrophy.

\section{Results}

\section{Tau isoforms in mouse lemurs}

In humans, MAPT gene can produce a variety of isoforms by alternative splicing. In normal adult human brain there are six isoforms that differ by sequences from exons 2 and 3 that encode $\mathrm{N}$-terminal sequences, and exon 10 that encodes a microtubule binding repeat sequence. When this latter exon is present there are four microtubule binding repeats (4R-tau) and when absent there are three microtubule-binding repeats (3R-tau) [24]. In adult humans, all six brain isoforms are present leading to the presence of both $4 R$ and $3 R$ tau [15]. In adult wild-type mice, the $4 R$ tau is the only isoform [24]. Analysis of tau isoforms in mouse lemurs by immunoblots showed that mouse lemurs present with both the $4 \mathrm{R}$ and $3 \mathrm{R}$ isoforms, while the isoforms corresponding to exon 2 were not detected due to either their absence or differences in the protein sequence (Additional File 1: Fig. $\mathrm{S} 1 \mathrm{~b})$.

\section{Characterization and inoculation of human brain homogenates}

We prepared two brain homogenates from sporadic AD patients, with each homogenate consisting of a combination of four brain extracts from patients with either a slowly evolving form of $\mathrm{AD}$ (defined by a disease duration of 5 to 8 years (AD1)) or a rapidly evolving form of $\mathrm{AD}$ (defined by a disease duration of 6 months to 3 years (AD2)). A third "control" homogenate, was prepared from the brains of two non-demented individuals (Ctrl). The characteristics of the selected subjects are presented in Additional File 1: Table S1 and Additional File 1: Fig. S2. The amount of $A \beta$, tau and neuroinflammatory proteins differed slightly between the brain homogenates, as the AD2 brain extract displayed more total tau and phospho-tau181, but less $A \beta_{38}$ and $A \beta_{40}$ than the AD1 one (Additional File 1: Fig. S2g-l). Iba1 and GFAP levels were similar in the two AD homogenates (Additional File 1: Fig. S2m-o). Brain homogenates were bilaterally inoculated into the posterior cingulate cortex and 
underlying corpus callosum of young-adult 1.5-year-old mouse lemurs $(6.25 \mu \mathrm{l} / \mathrm{site}, \mathrm{n}=6 /$ group $)$. This corresponded to $33.75,34.56$ and $34.37 \mu \mathrm{g}$ of total proteins per site, respectively for the Ctrl, AD1 and AD2 groups. This corresponds to $0,0.75$ and $1 \mathrm{pg}$ of $\mathrm{A} \beta 42$ and $0,0.063$ and $0.125 \mu \mathrm{g}$ of phospho-tau181. As depicted in Additional File 1: Fig. S3 showing regions connected to the posterior cingulate, the needle tract passed through the medial part of the parietal cortex (Broadman area 7) [27].

\section{$A \beta$ pathology induction and spreading after $A D$ brain inoculation}

Mouse lemurs were euthanized at 21 months post-inoculation (mpi) and their brains were studied by histology. All of AD-inoculated animals $(n=12)$ developed $A \beta$ deposits (Figs. 1, 2, 3 and 4), whereas none of the Ctrl-inoculated animals displayed any $A \beta$ pathology $(n=6$, Fig. $5 a-b)$. These deposits were detected using 4G8 (Fig. 1a, c, g-h) or A 342 (Fig. 1b, d) antibodies as well as a Thioflavin $\mathrm{S}$ staining (Fig. 1e-f). They occurred in the forms of diffuse (Fig. 1a-b) and dense (Fig. 1c-f) parenchymal $A \beta$ plaques or $A \beta$ angiopathy affecting cortical and hippocampal vessels (Fig. 1g-h), although angiopathy was less prominent than parenchymal deposits. $A \beta$ pathology spread widely throughout the brain and was detected in most cortical regions (Figs. 2, 3, 4a, c-f). Indeed, animals from both $\mathrm{AD}$-inoculated groups showed $\mathrm{A} \beta$ deposition in the inoculated posterior cingulate cortex (Figs. 2i, 3a) and/or around the needle tract in the parietal cortex (area 7) (Figs. 2l-n, r, 3b). Adjacent regions including the retrosplenial cortex (Figs. 2q, 3c), anterior cingulate cortex (Figs. 2a, e, 3d) and parietal area 5 (Figs. 2j-k, 3e) also displayed $A \beta$ pathology. Additionally, $A \beta$ plaques were detected in the hippocampus (Figs. 2p, t, 3f) and other regions more distant from the inoculation sites including the superior temporal cortex (areas 22, 21 and 20) (Figs. 2g, o, s, 3g), the parietal cortex involving the primary somatosensory cortex (areas 1-3, Fig. 2c, f) and the frontal cortex, including the primary motor cortex (area 4, Figs. 2b, 3h). Occipital areas also displayed $A \beta$ deposits (area 17, Fig. $2 \mathrm{u}-\mathrm{v}$; area 18, Fig. $2 \mathrm{w}-\mathrm{x}$ ). A $\beta$ pathology was not detected in the entorhinal cortex (Fig. 3i) or in deep grey nuclei, brainstem or cerebellum. Quantitative analysis did not reveal any difference between the two AD-inoculated groups (Fig. 3).

\section{Tau pathology induction and spreading after AD brain inoculation}

All AD-inoculated lemurs developed intraneuronal tau accumulations resembling neurofibrillary tangles (NFTs, Fig. 6a-d) and neuropil threads (NTs, Fig. 6e-h), at the inoculation sites and in several other regions (Figs. 4b, g-j, Figs. 7, 8 and 9). Conversely, Ctrl-inoculated animals did not display any tau pathology (Fig. 5c-d). In ADinoculated animals, tau pathology was detected using AT8 that detects phosphorylation of S202 and T205 [38] (Figs. 6, 7), AT100 that detects phosphorylation at T212 and at S214 that is phosphorylated in AD brains but not in normal brains [38] (Fig. 6d, g), an anti-pS422 antibody (phosphorylation at S422 occurs in AD but not in normal brains [38], Fig. 6c, f), and Gallyas silver staining (Fig. 6h). Neuritic plaques were not detected in any animal. AT8positive NFTs were localized at the level of the inoculation site (posterior cingulate cortex (Figs. 7i, 8a), parietal area 7 (Figs. $7 \mathrm{~m}, 8 \mathrm{~b}$ ) as well as in juxtaposing regions (retrosplenial cortex (Figs. 7n, q, s, 8c), anterior cingulate cortex (Figs. 7a, c, 8d), parietal area 5 (Figs. 7e, 8e)). They were also detected within the hippocampus (Figs. 7k, o, r, 8f) and temporal area 22 (Fig. 7g). Although temporal regions such as area 21 was never involved, some regions more distant from the inoculation sites displayed NFTs (temporal area 20 (Figs. 7l, p, t, 8g), area 13-16, parietal areas 1-3, frontal area 4 (Fig. $8 \mathrm{~h}$ ) and entorhinal cortex (Figs. 7h, 8i)). Occipital regions (areas 18-17) as well as the most frontal regions did not display tau-positive NFTs, despite the presence of A $\beta$ deposits (Fig. 4). AT8positive neuropil threads were mainly localized at the inoculation sites (posterior cingulate cortex (Figs. 7i, 8a), corpus callosum (Figs. 7f, j, Fig. 9b), but were barely present around the needle tract (area 7, Figs. 7m, 9c). They were also induced to a lesser degree in juxtaposing regions (retrosplenial cortex (Figs. 7n, 9d), anterior cingulate cortex (Fig. 9e), parietal area 5 (Figs. 7e, 9f)) and in distant regions (temporal area 20 (Figs. 7p, 9g), entorhinal cortex (Fig. 9h) and amydgala (Fig. 7b, d). Except for one or two animals, neuropil threads were not detected in the hippocampus (Fig. 9i) nor in the frontal cortex (area 4, Fig. 9j). As was the case for $A \beta$, quantitative analysis did not reveal any difference in tau pathologies between the two AD-inoculated groups (Figs. 8 and 9).

\section{Similar microglial response in AD- and Ctrl-inoculated animals}

Neuroinflammation was assessed using a histological marker for activated microglia (HLA-DR). Reactive microglia were observed both in the parenchyma (Fig. 10a) and around the vasculature (Fig. 10b) of ADand Ctrl-inoculated animals. Microglial response mainly involved the inoculation sites (Fig. 10c-e) and was not detected in other regions, even in the presence of $A \beta$ plaques. We did not detect any differences between the groups, suggesting that the inoculation of AD-brain extracts does not induce an exacerbated neuroinflammatory response in comparison with human Ctrl-brain extract. 

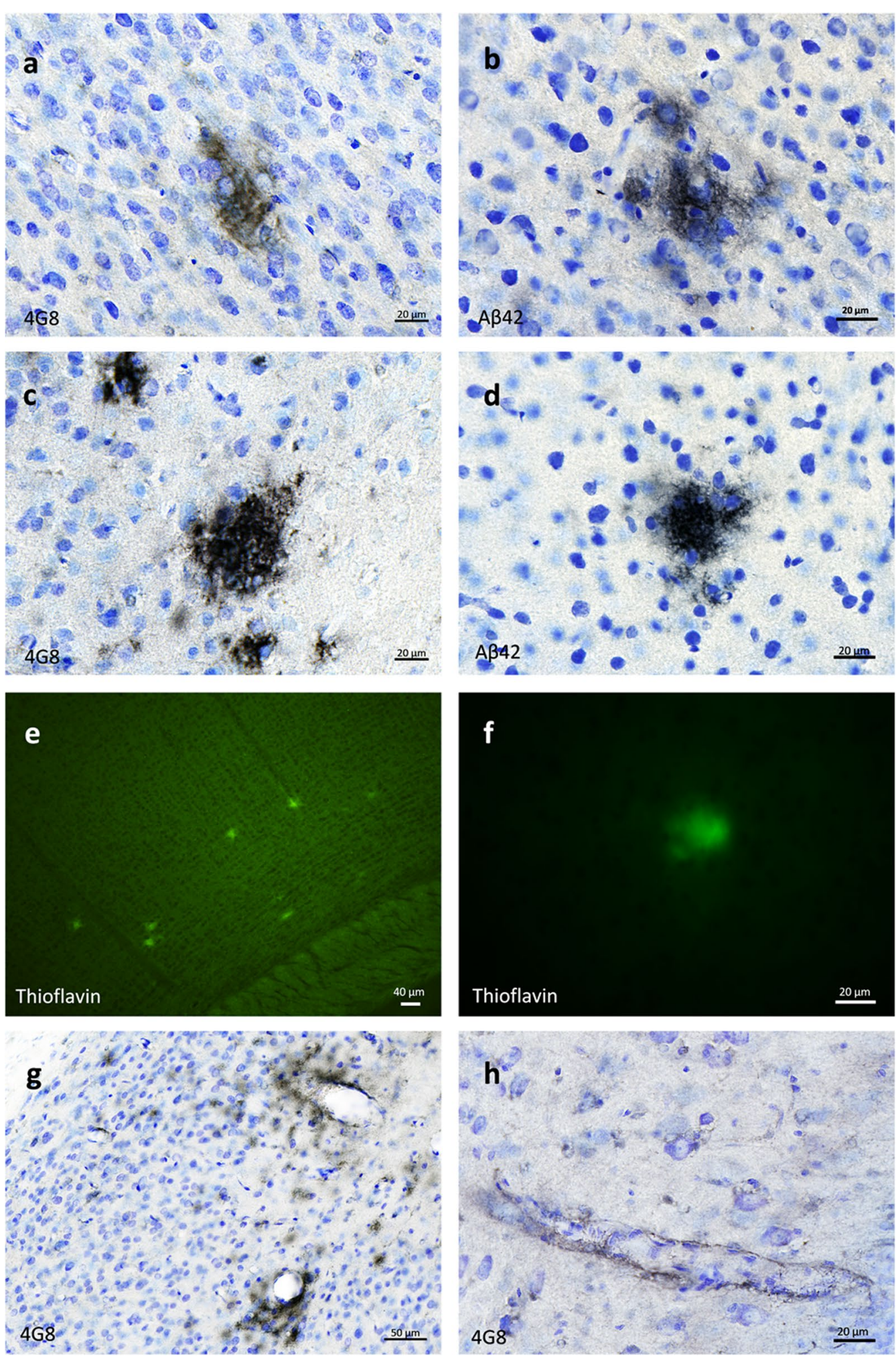

Fig. $1 A \beta$ plaques and vascular deposits in AD-inoculated mouse lemurs. Parenchymal diffuse $\mathbf{a}-\mathbf{b}$ and dense $\mathbf{c}-\mathbf{d}$ plaques stained by $4 \mathrm{G} 8$ or $A \beta 42$ antibodies in AD-brain inoculated mouse lemurs. Plaques were also Thioflavin S-positive $\mathbf{e}-\mathbf{f}$. Cerebral A $\beta$ angiopathy was also observed in cortical regions $\mathbf{g}$ and in the hippocampus $\mathbf{h}$ (4G8 antibody). Scale bars: $20 \mu \mathrm{m}$ in a-d, f, h; $40 \mu \mathrm{m}$ in e; $50 \mu \mathrm{m}$ in $\mathbf{g}$ 


\section{Cognitive deficits induced by AD brain inoculation}

Learning and long-term memory capabilities were evaluated using discrimination tasks in a jumping stand apparatus [31] before inoculation and at 4, 9, 15 and $21 \mathrm{mpi}$. The jumping stand apparatus was designed to test the cognition of mouse lemurs using discrimination tasks while taking into account their arboreal lifestyle as well as their sensorial and behavioral skills. At each time-point, a new pair of visual stimuli was introduced to the animals, and learning abilities were evaluated as the lemur had to identify the positive stimulus to reach its nesting box (Additional File 2). Long-term memory was evaluated through the recall of the discrimination task learned during the previous session, 4 to 6 months earlier. At the final timepoint, i.e. at $21 \mathrm{mpi}$, and following a successful discrimination learning session, a reversal learning test was performed to evaluate cognitive flexibility.

AD-inoculated animals showed lower learning abilities in comparison with Ctrl animals (Fig. 11a). Indeed, for the first discrimination task, before the inoculation of the brain extracts (M0), all groups required a similar number of trials to learn the rewarded stimulus $(15.3 \pm 5.8$, $16.8 \pm 8.6$ and $14.5 \pm 7.4$ trials for Ctrl, AD1 and AD2 inoculated animals, respectively). Learning performances improved in the Ctrl-inoculated group, reaching the best possible score (at least 8 correct choices out of the first 10 consecutive trials) as early as during the second learning session at $4 \mathrm{mpi}$ (M4), thus demonstrating highly effective acquisition of the learning set. Conversely, learning abilities in the two AD groups declined markedly over time, and performances were significantly worse compared to the Ctrl group throughout the follow-up (Fig. 11a, $p$ $=0.0002$ and $p=0.004$, respectively for AD1 and AD2). Long-term memory performance also differed between the groups throughout the study, but no post-hoc differences were detected between AD-inoculated animals and controls (Fig. 11b). A reversal learning test was performed only at $21 \mathrm{mpi}$ and Ctrl animals performed better than AD1 or AD2-inoculated lemurs (Fig. 11c, $p=0.024$ and $p=0.0045$, respectively). For all of the evaluated cognitive functions, no statistical difference was observed between AD1- and AD2-inoculated lemurs (Fig. 11a-c). Altogether, these data suggest that inoculation with $\mathrm{AD}$ brain extracts impairs learning abilities as well as cognitive flexibility, while long-term memory is globally preserved. Additionally, motor function was evaluated and showed that, as expected, all animals displayed similar motor skills all throughout the follow-up (Fig. 11d). These data suggest that cognitive deficits observed in AD-inoculated animals in the jumping stand apparatus were not related to motor impairments.

\section{Progressive cerebral atrophy induced by AD brain inoculation}

Brain MRI acquisitions were performed before inoculation and at 4, 9, 15 and $21 \mathrm{mpi}$. To increase statistical power, AD1 and AD2-inoculated animals were grouped within a unique AD group to be compared with Ctrlinoculated lemurs. Cerebral atrophy was evaluated using an automated voxel-based morphometry analysis [36]. To control for multiple comparisons, an adjusted p-value was calculated using the voxel-wise false discovery rate (FDR-corrected $p<0.05$ ), with extent threshold values of 10 voxels. At $4 \mathrm{mpi}$, only a slight atrophy was detected in the inoculated posterior cingulate cortex of $\mathrm{AD}$ animals, compared to the Ctrl group (Fig. 12a). This atrophy did not progress between 4 and 9 mpi (Fig. 12b), but between 9 and $15 \mathrm{mpi}$ significant bilateral atrophy occurred in several other brain regions (Fig. 12c). It involved the inoculation site, i.e. the posterior cingulate cortex, and the parietal cortex (area 7, site with the needle tract) of AD brain-inoculated lemurs (Fig. 12c). Grey matter loss was also observed close to the inoculation sites in the retrosplenial, parietal area 5 and anterior cingulate cortices (Fig. 12c). In addition, cerebral atrophy was reported in several other cortical areas including the prefrontal cortex (antero-medial area), frontal cortex (superior frontal cortex, areas 4 and 6), parietal areas 1-3, temporal cortex (entorhinal and periamygdalar cortices), insular cortex (areas 13-16), and occipital visual cortex (areas 18 and 17) (Fig. 12c). Atrophy was also detected in some subcortical regions such as the hippocampus, the basal forebrain (including the diagonal band of Broca and nucleus accumbens), claustrum, septum, basal ganglia (including the caudate nucleus and putamen) and medial thalamus (Fig. 12c). Atrophy did not further increase from 15 to 21 months post-inoculation (Fig. 12d).

\section{(See figure on next page.)}

Fig. $2 A \beta$ pathology throughout the brains of mouse lemurs inoculated with AD brain extracts. Representative images of $4 \mathrm{G} 8$ immunolabelling showing $A \beta$ pathology throughout the brains of mouse lemurs following $A D$ brain extracts inoculations. A $\beta$ deposition was observed in the inoculated posterior cingulate cortex (i) and around the needle tract in the parietal cortex (area $7 ; \mathbf{I}-\mathbf{n}, \mathbf{r}$ ). Adjacent regions including the retrosplenial cortex $(\mathbf{q})$, parietal area $5(\mathbf{j}-\mathbf{k})$ and anterior cingulate cortex $(\mathbf{a}, \mathbf{e})$ also displayed A $\beta$ pathology. Additionally, A $\beta$ plaques were detected in the hippocampus $(\mathbf{p}, \mathbf{t})$ and other regions more distant from the inoculation sites including the superior temporal cortex (areas 22 and $21 ; \mathbf{g}, \mathbf{o}, \mathbf{s}$ ), the parietal areas 1-3 (c, $\mathbf{f}$ ) and the frontal cortex (area 4; b). Occipital areas also displayed A 3 deposits (areas 17 and 18; $\mathbf{u}-\mathbf{x}$ ). The red crosses indicate the inoculated region. Scale bars: $500 \mu \mathrm{m}$ in whole slice images, $100 \mu \mathrm{m}$ in zooms and $20 \mu \mathrm{m}$ in inserts 


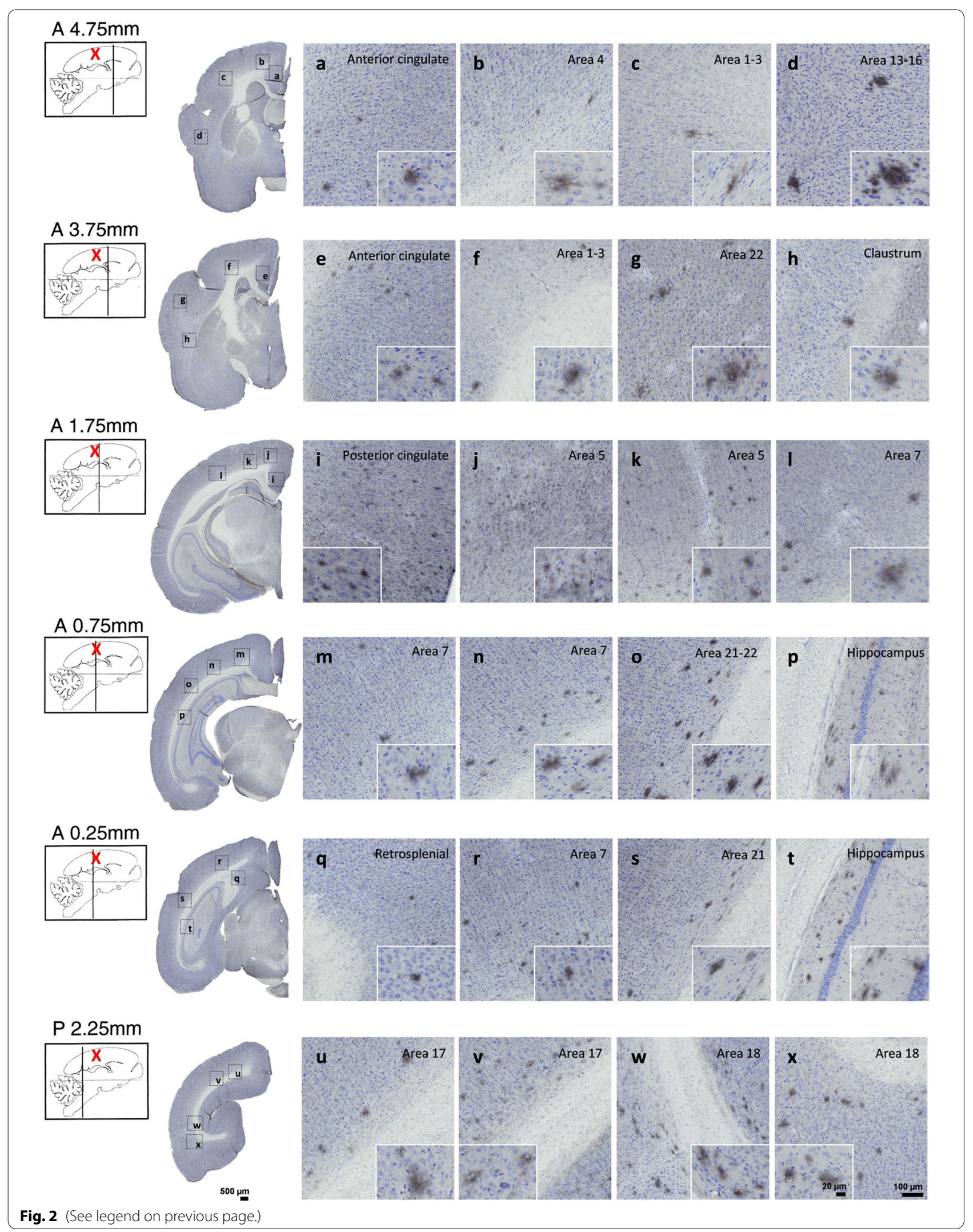




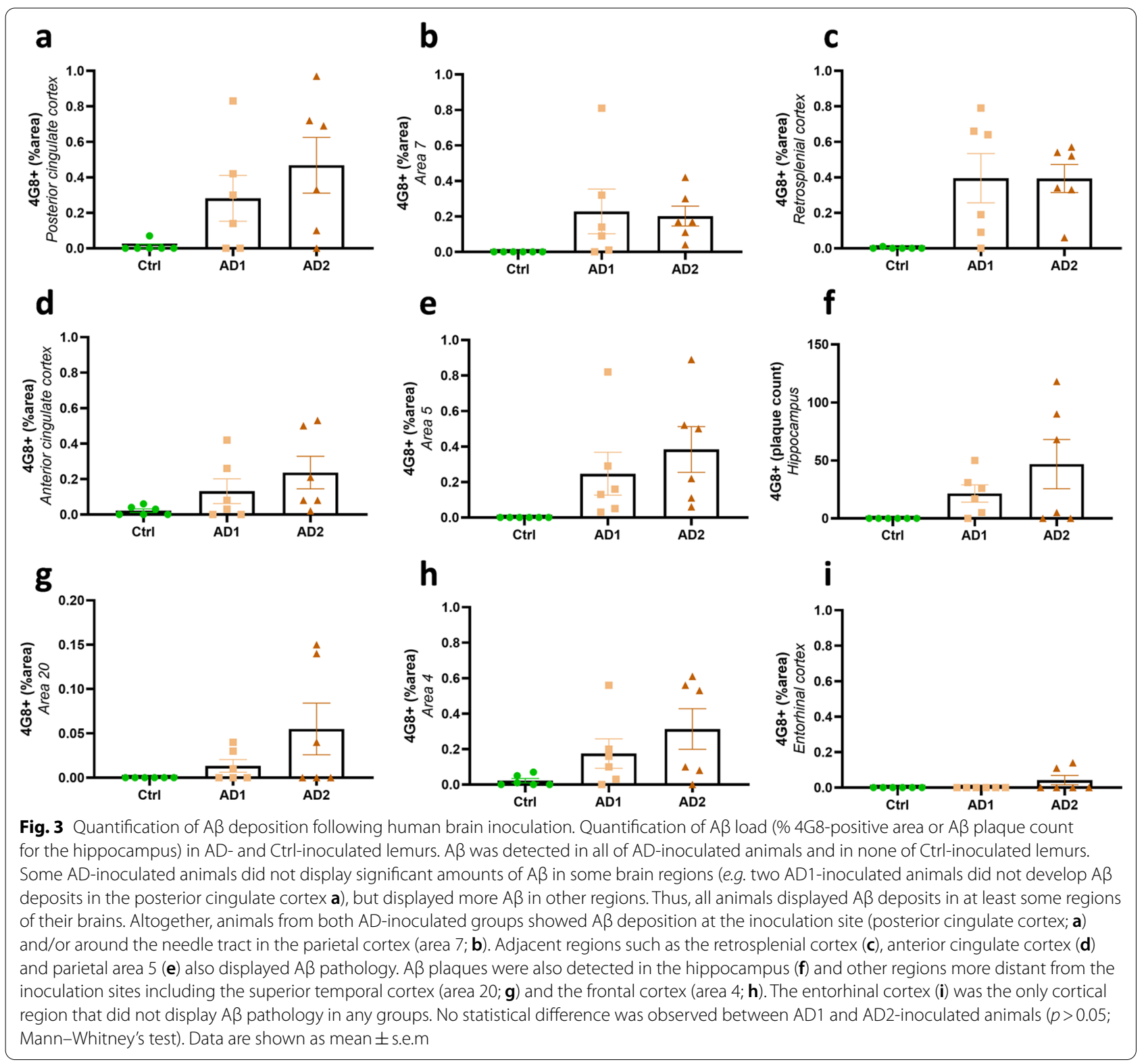

\section{Discussion}

For the first time in a primate, we induced widespread $\mathrm{A} \beta$ and tau pathologies along with cognitive impairments and cerebral atrophy following the focal inoculation of $\mathrm{AD}$ brain extracts in the cingulate cortex and underlying corpus callosum. These results were replicated using two different batches of AD brain extracts.

$A \beta$ deposits were detected using specific antibodies as well as Thioflavin S staining. They were observed close to the inoculation site and in almost all cortical regions (except for the entorhinal cortex) as well as in the hippocampus, suggesting their efficient spreading within the whole brain. Tau-positive pathology occurred in the forms of neurofibrillary tangles and neuropil threads while neuritic plaques were not detected. They were detected using several antibodies (AT8, AT100, ps422) and Gallyas staining. Tau pathology was evident at the inoculation site and throughout the brain. However some brain regions that were relatively close to the inoculation sites were spared, such as temporal (area 21) and occipital regions (areas 18 and 17). This suggests that the spatial progression of tau did not occur solely via a systematic isotropic diffusion from the injection site to proximal regions. Interestingly, regions such as the entorhinal cortex, that are connected to the cingulate cortex [29] and are distant from the inoculation site were tau-positive but 


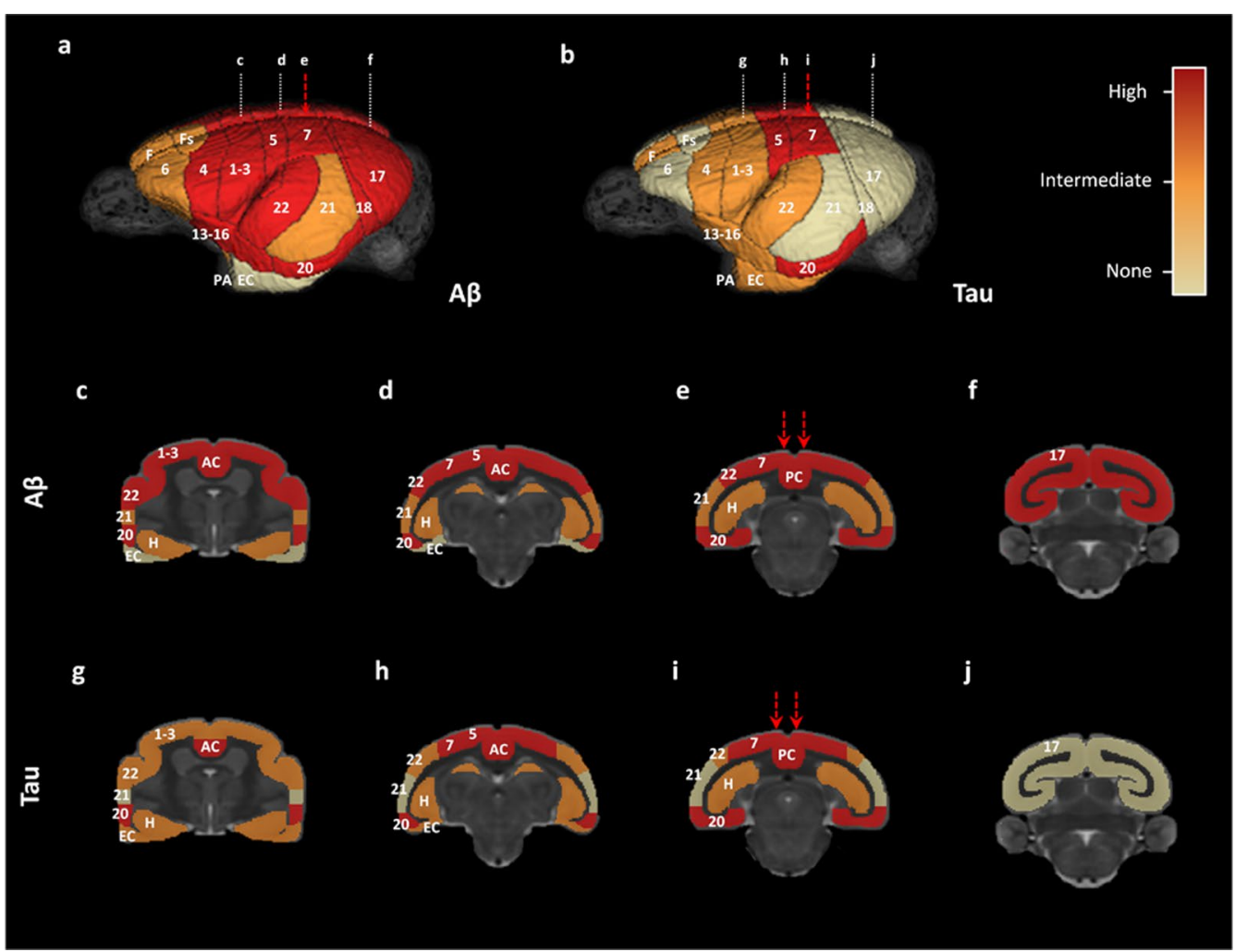

Fig. 4 Overview of $A \beta$ and tau pathology spreading throughout the brains of mouse lemurs inoculated with AD brain extracts. Three-dimensional rendering of $A \beta$ and tau pathologies in mouse lemur brains using a three-level semi-quantitative scale (no lesions/intermediate/high lesion load). (a, c-f) A $\beta$ pathology involved almost all cortical regions, except for the entorhinal cortex. The hippocampus was also A $\beta$-positive. (b, g-j) High levels of tau lesions were reported at the inoculated sites (posterior cingulate cortex, area 7) as well as in juxtaposing regions such as the area 5. Distant regions such as the area 20 also displayed high tau pathology. The hippocampus, entorhinal cortex, and other cortical areas displayed intermediate tau lesion loads. The red arrows indicate the needle tracts. Numbers represent Brodmann areas as reported in the mouse lemur brain by (Le Gros Clark, 1931). AC: anterior cingulate cortex, EC: entorhinal cortex, F: antero-medial frontal cortex, Fs: superior frontal cortex, H: hippocampus, PA: peri-amygdalar cortex, PC: posterior cingulate cortex

A $\beta$-negative. This suggests that tau pathology occurred, at least in part, through a transit along neuroanatomical pathways and following different routes as compared to $A \beta$. Importantly, both $A \beta$ and tau pathologies were detected after a relatively short incubation period of 21 months in young-adult 1.5-year-old primates that are typically devoid of any lesions at this age. We speculate that aggregate deposition might have started even earlier since cerebral atrophy was detected between 9 and 15 mpi.

A chronic neuroinflammatory response, evaluated by HLA-DR staining, was detected at the inoculation sites after human brain extracts inoculation. Such inflammation was not observed in non-inoculated animals (data not shown). Here, inflammation was restricted to the cingulate cortex and underlying corpus callosum, while $A \beta$ and tau pathologies were widespread in the brains of AD-inoculated animals. Also, no difference in microglial activation was observed between the groups of AD- or
Ctrl-inoculated animals. This suggests that neuroinflammation cannot explain most of the differences between AD- and Ctrl-inoculated animals. However, one cannot exclude that subtle changes in cell morphology or differences in secreted mediators reflecting different stages of activation could have occurred in AD- and Ctrl-inoculated animals.

Many questions remain following the several neuropathological observational studies in humans showing that $A \beta$ pathology is transmissible [17]. In particular, it is critical to assess whether tau pathology can also be iatrogenically transmitted. Only one recent article suggested that tau could also be detected in patients with iatrogenic $\mathrm{A} \beta$ pathology [16]. However this study could not answer if the tauopathy was transmitted or was a consequence of $\mathrm{A} \beta$ pathology. Experimental studies in transgenic mouse models have suggested that tau can be transmitted [6], but these models mainly rely on tau protein overexpression. Here, we show definitively that tau pathologies can 

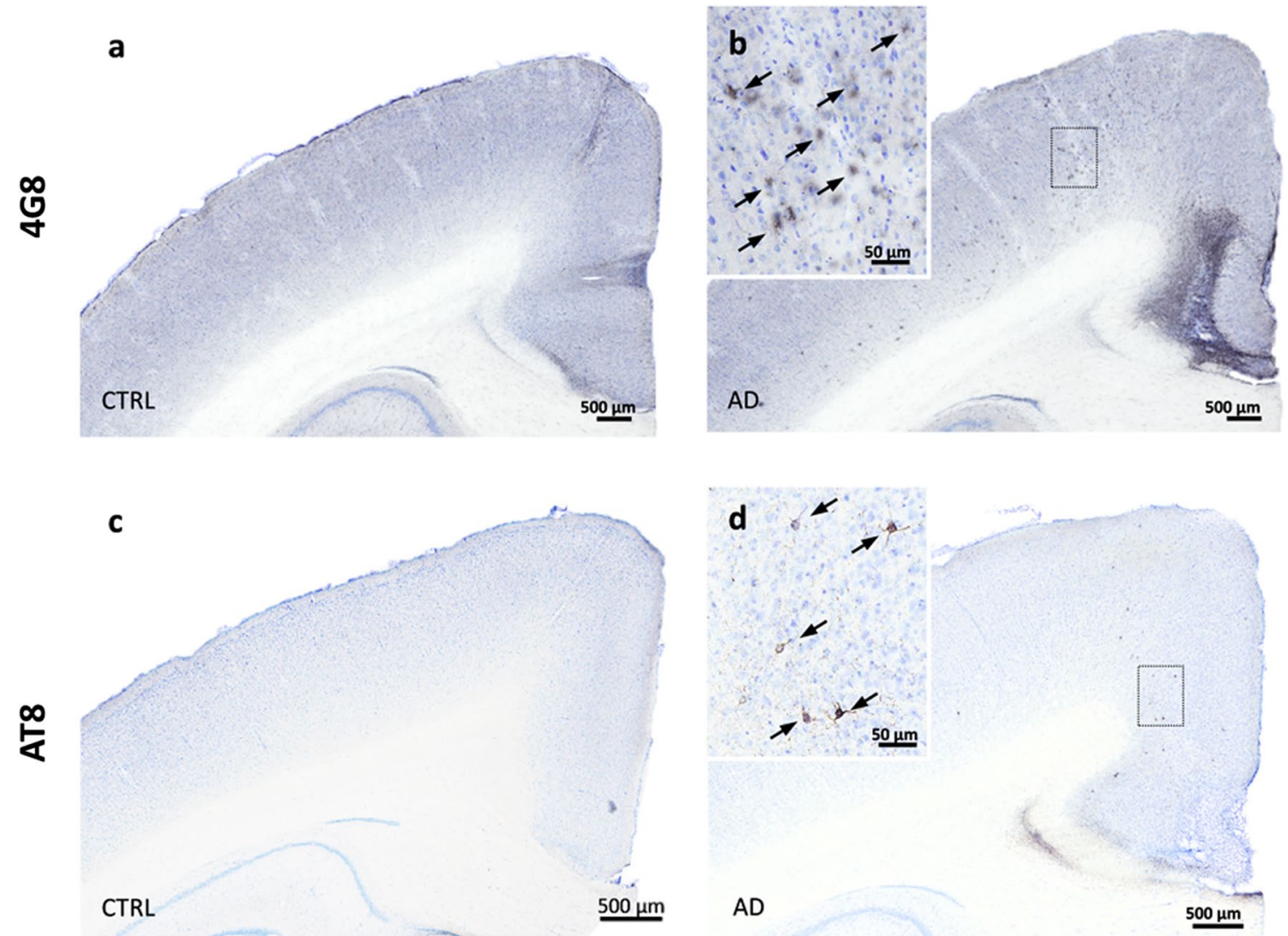

Fig.5 $A \beta$ and tau pathologies were not induced following Ctrl-brain extract inoculations. Representative sections of $4 \mathrm{G} 8$ (a-b) and AT8 (c-d) stainings in mouse lemurs inoculated with the control brain extract $(\mathbf{a}, \mathbf{c})$ compared with AD-inoculated animals (b, $\mathbf{d})$. No A $\beta$ and tau deposits were observed in control animals $(\mathbf{a}, \mathbf{c})$ while $A \beta(\mathbf{b})$ and tau $(\mathbf{d})$ were detected in $A D$-inoculated animals. Scale bars: $500 \mu m$

be transmitted in a primate expressing physiological levels of endogenous tau proteins. Staining for tau pathology was well marked and not necessarily in the direct vicinity of $A \beta$ pathology nor in the same regions. At least three different hypothesis could explain this tau transmission. First, tau seeds from the inoculated brain extracts were responsible for the induction of the tau pathology. This hypothesis is consistent with the fact that brain extracts can induce tau pathologies in mice $[7,11]$ and that immunodepletion for tau suppresses tau pathology induction [7]. The second hypothesis is that $A \beta$ contained in the human brain extracts (possibly oligomeric forms of $A \beta$ ) induced tau pathology in mouse lemur's brain in addition to the induction of $A \beta$ pathology. Thus, tau pathology would not occur through a direct seeding effect of the inoculated human-tau pathology. The lack of co-localization of $\mathrm{A} \beta$ plaques (that are known to be also a reservoir for $A \beta$ oligomers) and tau lesions does not support this hypothesis. The third hypothesis is that the inoculated brain extracts contained an undetected compound other than $A \beta$ or tau that induced tau pathology. While we can not rule out this hypothesis, it seems less likely than the first one.

Previous studies have evaluated $A \beta$ and tau pathology induction in primates following the inoculation of $A D$ brain extracts. A 3.5 to 7-year-long study conducted on marmosets intracerebrally inoculated with large volumes of AD brain extracts ( $300 \mu \mathrm{l}$ of $10 \%$ homogenates distributed within 6 inoculation sites) led to moderate $A \beta$ deposition in most animals but not to tau pathology nor to other AD-like pathological features [2, 23, 34]. In a previous study, our group inoculated $\mathrm{AD}$ brain extracts in the hippocampus and overlying cortex of mouse lemurs, leading to sparse $A \beta$ and tau deposits close to the inoculation site [11]. The more severe $A \beta$ and tau pathological inductions observed in the present study, after inoculation of a relatively small volume of brain extracts $(25 \mu \mathrm{l})$, could in part be explained by variations in brain extract preparations and injection protocols. Here, we sonicated each sample prior to the inoculation as this procedure was shown to enhance the seeding and spreading capacities of brain extracts, supposedly by increasing the level 

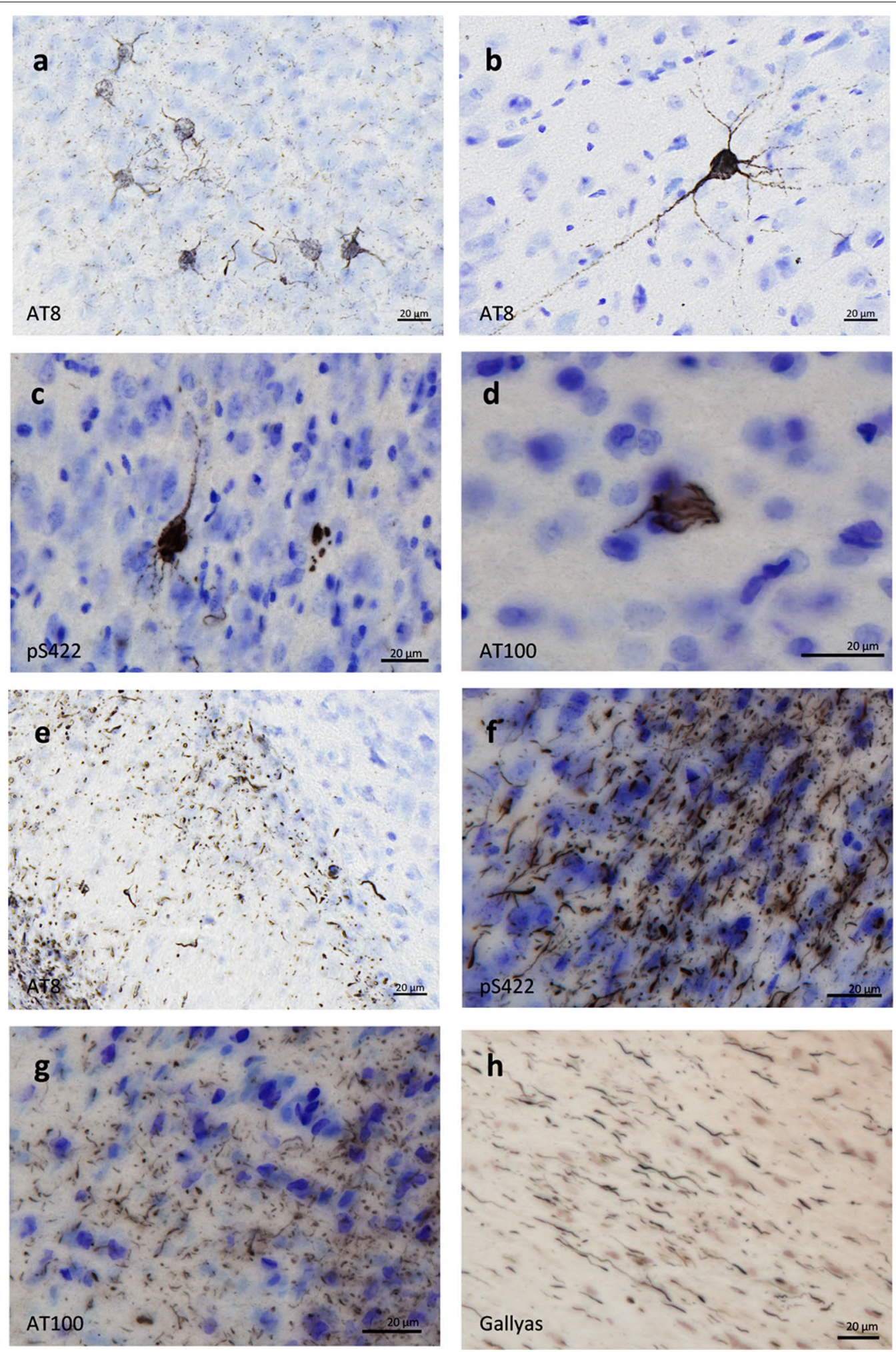

Fig. 6 Intraneuronal neurofibrillary tangle and neuropil threads in AD-inoculated animals. AT8-positive neurofibrillary tangle in AD brain inoculed in mouse lemurs were detected after staining with AT8 (a-b), an antibody detecting phosphorylation of S422 (c), and another one targeting phosphorylation of T212 and S214 (AT100) (d). Neuropil threads were revealed after staining with AT8 (c), anti-pS422 antibody (f), AT100 (g), and Gallyas staining (h). Scale bars: $20 \mu \mathrm{m}$ 
of molecular interfaces for templated misfolding and generating smaller $A \beta$ and tau assemblies that can more readily propagate through the brain than large fibrils [20]. Obviously, $A \beta /$ tau-positive samples that contaminated humans (e.g. cadaver-sourced human growth hormones or dura matter) were not sonicated, which could explain much longer incubation times in iatrogenic transmission cases. In addition, in the present study, the injections were performed into highly connected brain areas, such as the corpus callosum and the posterior cingulate cortex. The former is indeed the major commissural tract connecting both hemispheres and the latter is a functional connectivity hub in mouse lemurs [10]. Moreover, the cingulate cortex is involved in the default-mode network, which is disrupted and highly vulnerable to $A \beta$ deposition in $\mathrm{AD}$ [5]. Additionally, it is one of the first regions to display atrophy in the course of $\mathrm{AD}$ [3].

Whether $A \beta$ and tau iatrogenic transmissions can be associated with clinical impacts in humans remains to be elucidated. Here, along with neuropathological lesions in their brains, AD-inoculated animals displayed cognitive impairments and bilateral brain atrophy. In humans, NFT deposition has been associated with clinical decline [28]. Here, even if we cannot conclude that there is a direct link between $A \beta$ and tau pathologies and cognitive deficits, the occurrence of cognitive alterations suggests that exogenous exposure to misfolded $A \beta$ and tau seeds can translate into clinical manifestations. This is in line with our previous study showing that $A D$ brain extract inoculation in mouse lemurs leads to cognitive impairments despite sparse $\mathrm{A} \beta$ and tau deposition [11]. Altogether, this raises concerns about the potential cognitive consequences in patients exposed to contaminated compounds, including vials of human cadaver-derived growth hormones that were shown to contain both $A \beta$ and tau seeds [9, 32].

Cerebral atrophy was prominent close to the inoculation site, i.e. the posterior cingulate, where it was first detected at 4 months post-inoculation. Atrophy worsened mainly from 9 to 15 months post-inoculation. This course of atrophy evolution suggests that grey matter loss was not related to an acute toxic effect of the inoculation. Atrophy also gradually spread to several other regions in AD-inoculated animals. Atrophy of the posterior cingulate cortex and connected prefrontal regions [39] and basal ganglia [19] is consistent with deficits in object discrimination learning and cognitive flexibility in $\mathrm{AD}$ brain extract-inoculated lemurs, as these structures are thought to play a critical role in instrumental learning and shifting abilities [30, 33, 35].

In conclusion, we provided the first experimental evidence of the transmission of an AD-like phenotype in a non-human primate that includes $A \beta$ and tau pathologies as well as cognitive impairments and cerebral atrophy. This first demonstration of the transmission of tau pathology in a primate calls for further studies in humans to assess the transmission of tau in subjects exposed to contaminated cadaver-derived compounds and surgical material. Our experimental study also outlined the possible consequences of $A \beta$ and tau transmissions in terms of cognitive impairments and cerebral atrophy. Evaluating these aspects of the pathology in patients at risk for $\mathrm{A} \beta$ and tau iatrogenic transmission through a systematic monitoring of $\mathrm{AD}$ biomarkers is thus urgently needed. Finally, our study also suggests that $\mathrm{AD}$ brain-inoculated mouse lemurs are highly relevant models to explore $\mathrm{AD}$ pathophysiology and ensure a greater translation of preclinical studies to patients.

\section{Materials and methods \\ Mouse lemurs}

Mouse lemurs were reproduced in an approved breeding center (UMR 7179 CNRS/MNHN, France; European Institutions Agreement \#962,773) and housed in our laboratory (Commissariat à l'Energie Atomique, Fontenay-aux-Roses center; European Institutions Agreement \#B92-032-02). Animals were housed individually in enriched cages containing a wooden nesting box and equipment allowing them to climb and jump freely. The environment was maintained at a constant temperature of $24-26{ }^{\circ} \mathrm{C}$ with a relative humidity of $55 \%$. The photoperiodic regime was based on a biannual alternation of long and short days (14 h of light at 250-350 lx/10 h of darkness in the summer period, $10 \mathrm{~h}$ of light at 250 $350 \mathrm{~lx} / 14 \mathrm{~h}$ of darkness in the winter period) in order to artificially reproduce the seasonal rhythm of the animals.

(See figure on next page.)

Fig. 7 Tau pathology throughout the brains of mouse lemurs inoculated with AD brain extracts. Representative images of AT8 immunolabelling showing tau pathology following AD brain extract inoculations in mouse lemurs. NFTs were observed at the level of the inoculation sites, e.g. in the posterior cingulate cortex (i) and the parietal area $7(\mathbf{m})$, as well as in juxtaposing regions such as the retrosplenial cortex (n, q, s), anterior cingulate cortex $(\mathbf{a}, \mathbf{c})$, parietal area $5(\mathbf{e})$. They were also detected within the hippocampus $(\mathbf{k}, \mathbf{o}, \mathbf{r})$ and temporal area 22 (g). Additionally, some regions more distant from the inoculation sites also displayed NFTs, e.g. the temporal area 20 (I, $\mathbf{p}$, t $)$ and the entorhinal cortex (h). Neuropil threads were mainly observed at the inoculation sites, e.g. in the corpus callosum $(\mathbf{f}, \mathbf{j})$ and posterior cingulate cortex (i). They were slightly induced around the needle tract (area $7 ; \mathbf{m})$, in juxtaposing regions such as the retrosplenial cortex (n), parietal area 5 (e) and in distant regions such as the temporal area 20 (p) and the amygdala $(\mathbf{b}, \mathbf{d})$. The red crosses indicate the inoculated region. Scale bars: $500 \mu \mathrm{m}$ in whole slice images, $20 \mu \mathrm{m}$ in zoomed images 

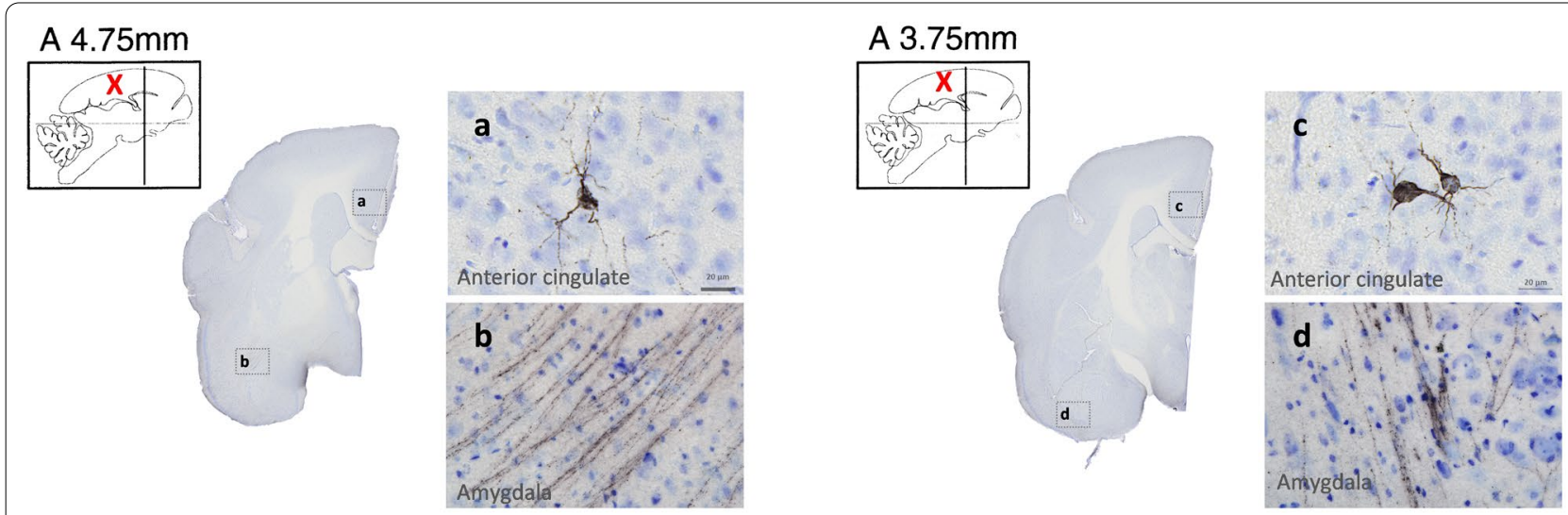

\section{A $1.75 \mathrm{~mm}$}
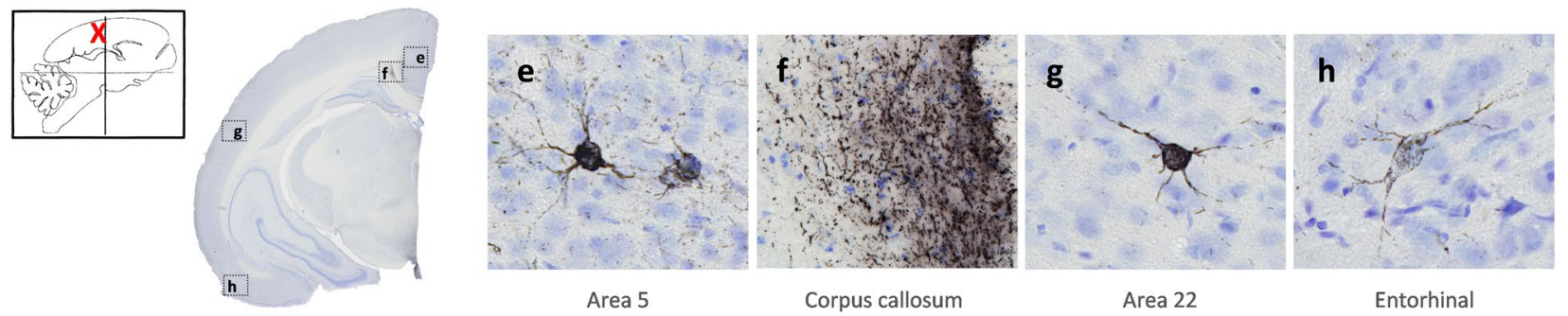

\section{A $1.00 \mathrm{~mm}$}
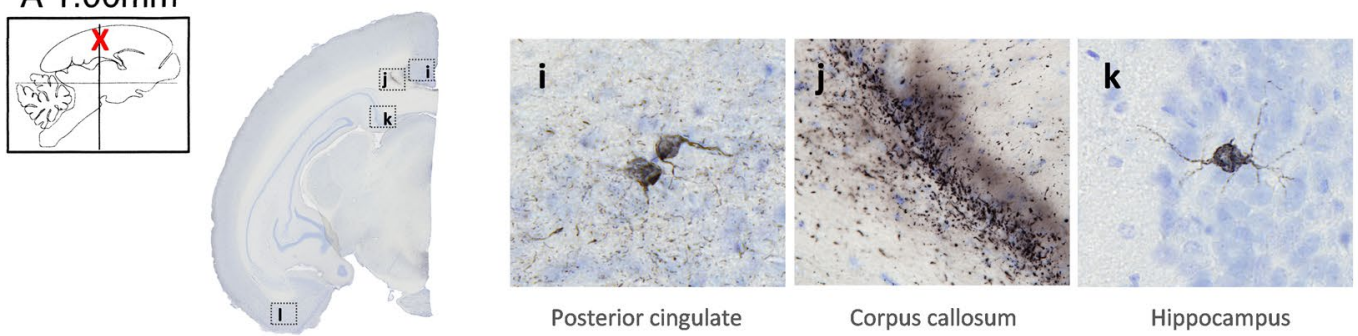

Posterior cingulate

Corpus callosum

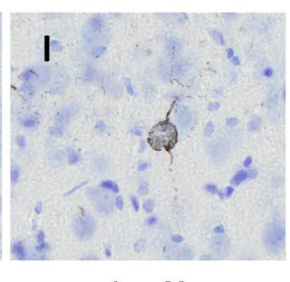

\section{A $0.25 \mathrm{~mm}$

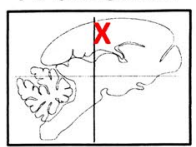

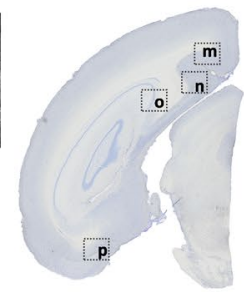
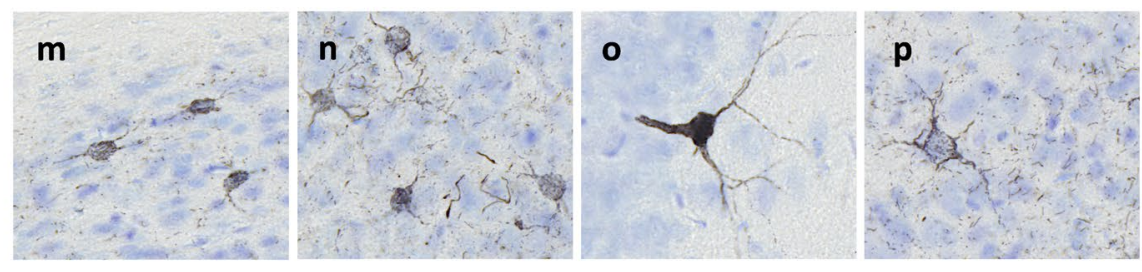

Area 7

Retrosplenial

Area 20
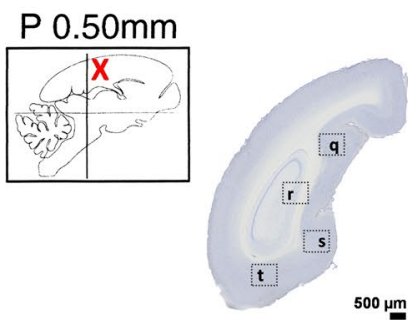

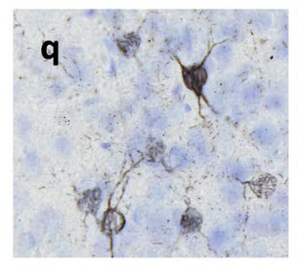

Retrosplenial

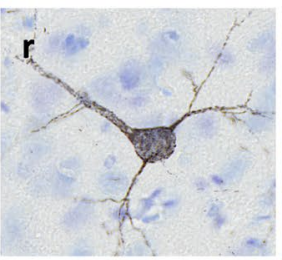

Hippocampus

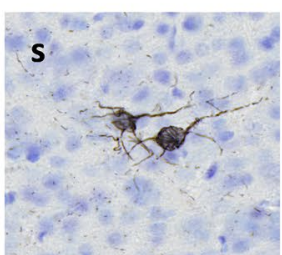

Retrosplenial

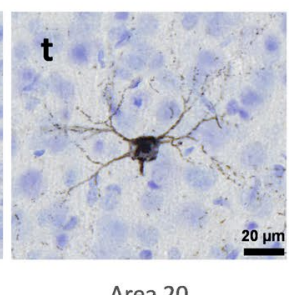

Area 20

Fig. 7 (See legend on previous page.) 

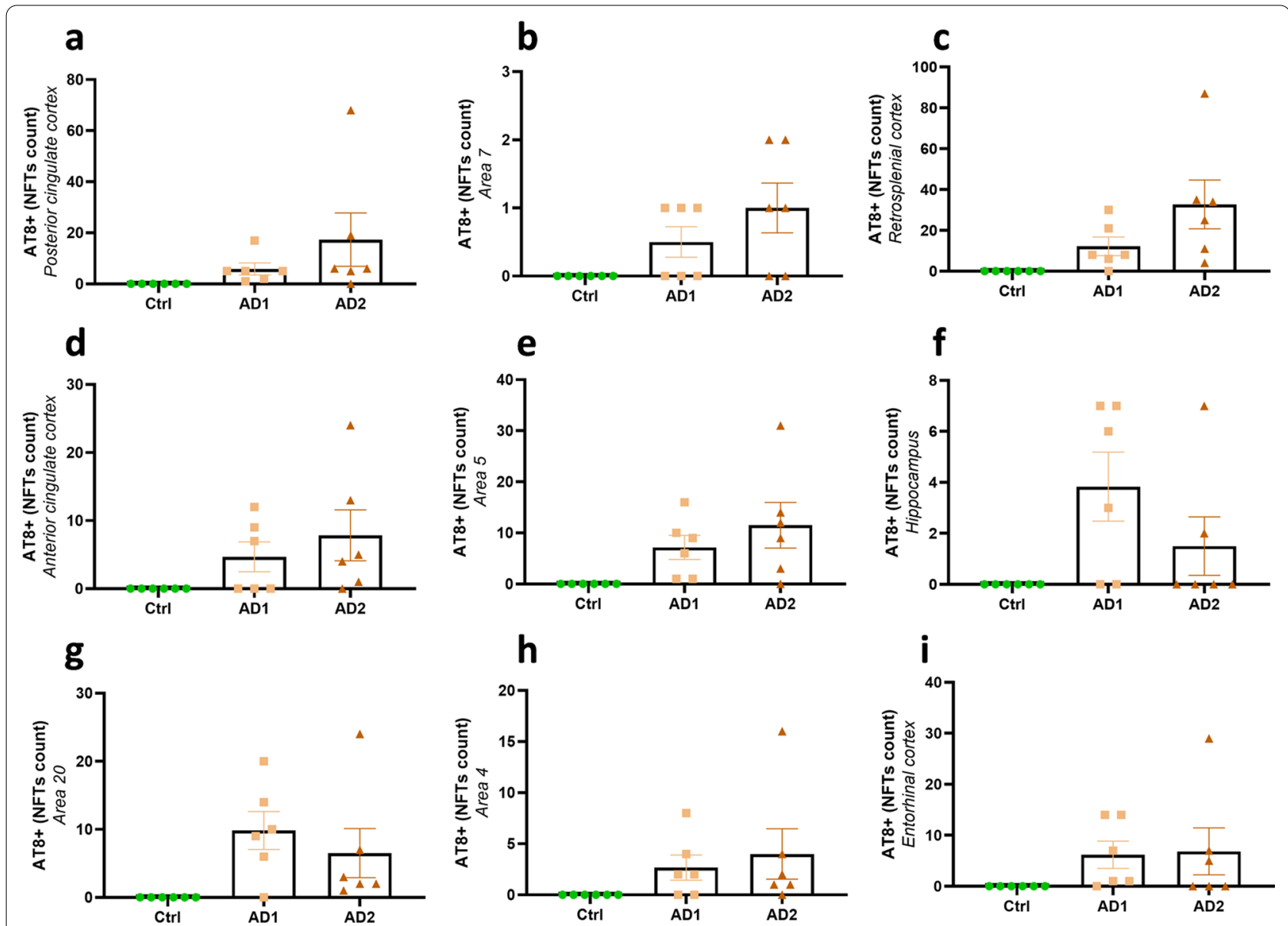

Fig. 8 Quantification of NFT pathology following human brain inoculation. Quantification of NFT burden (NFT count per region of interest) in AD- and Ctrl-inoculated lemurs. NFTs were detected in all of AD-inoculated animals and in none of Ctrl-inoculated lemurs. In AD-inoculated animals, NFT burden was increased at the level of the inoculation site, e.g. in the posterior cingulate cortex (a) and the parietal area 7 (b), as well as in juxtaposing regions, including the retrosplenial cortex (c), anterior cingulate cortex (d) and parietal area 5 (e). NFTs were also detected in the hippocampus (f) and in some regions more distant from the inoculation sites, including the temporal area $20(\mathbf{g})$, frontal area 4 (h) and entorhinal cortex (i). No statistical difference was reported between AD1- and AD2-inoculated lemurs ( $p>0.05$; Mann-Whitney's test). Data are shown as mean \pm s.e.m

Diet consisted of fresh fruits and a preparation based on cottage cheese, eggs, cereals and bananas. Water and food were available ad libitum.

Eighteen males were studied. In order to limit any agerelated modifications during the longitudinal follow-up, only young-adults (1.5-year-old) were included. Mouse lemurs were assigned to an experimental group in order to obtain cognitively comparable groups before the inoculations. After human brain inoculation, a 21-monthlong follow-up was conducted on the animals. Five other mouse lemurs were evaluated for characterization of tau isoforms by immunoblots. They were sacrificed due to various non-cerebral, non-experimental pathologies (age 9.7 to 10.5 years). All experimental procedures were performed in compliance with the European Union directive on the protection of animals used for scientific purposes (2010/63/EU). They were approved by a local ethics committee (CETEA-CEA DSV IdF) as well as by the French Ministry of Education and Research (authorization A17_083).

\section{Protein extraction and western blots for mouse lemur brains}

Hippocampi and cortices from five non-inoculated mouse lemurs were dissected and snap frozen in $1.5 \mathrm{~mL}$ tubes (Eppendorf). Tissus were homogenized in 10 volume of ice-cooled Tris-sucrose buffer (Tris- $\mathrm{HCl} 10 \mathrm{mM}$, $\mathrm{pH}$ 7.4 10\% sucrose added with protease inhibitor (Complete mini EDTA-free, Roche)) and were sonicated (20 pulses of $1 \mathrm{~s}$, amplitude $40 \%, 60 \mathrm{kHz}$ ) on ice. After $1 \mathrm{~h}$ 

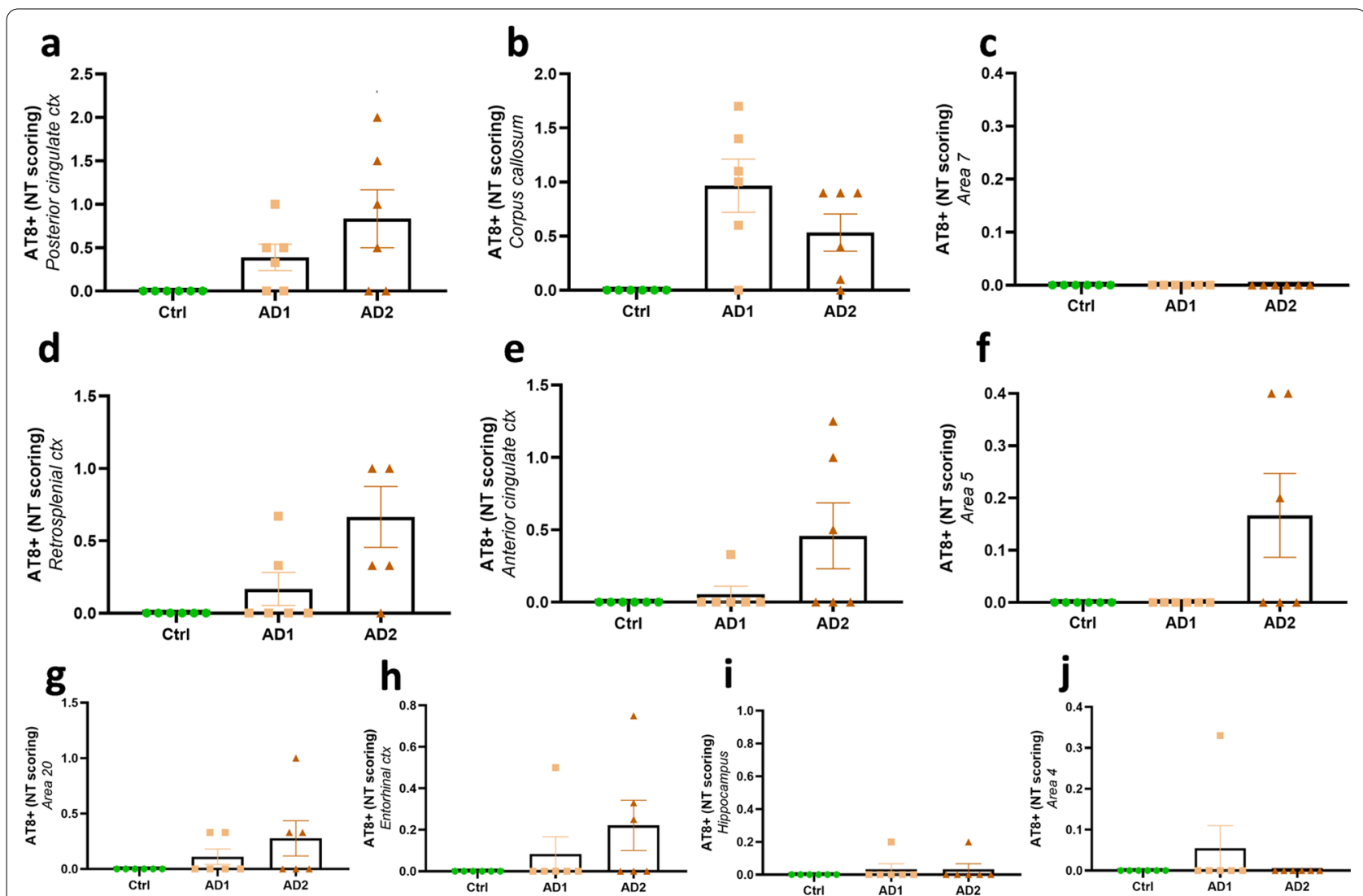

Fig. 9 Semi-quantitative scoring of neuropil thread pathology following human brain extracts inoculation. Neuropil threads were detected in AD-inoculated animals but not in theCtrl-inoculated lemurs. AT8-positive neuropil threads were mainly localized at the inoculation sites [posterior cingulate cortex (a) and corpus callosum (b)], but no lesion was observed around the needle tract (area 7; $\mathbf{c})$. They were also induced in juxtaposing regions, such as the retrosplenial cortex (d), anterior cingulate cortex (e), parietal area $5(\mathbf{f})$, and in distant regions such as the temporal area 20 (g) and entorhinal cortex (h). Except for one or two animals, neuropil threads were not detected in the hippocampus (i) nor in the frontal cortex (area 4; j). No difference was observed between the two AD-inoculated groups ( $p>0.05$; Mann-Whitney's test). Data are shown as mean \pm s.e.m

at $4{ }^{\circ} \mathrm{C}$, protein concentrations were determined using the BCA Protein Dosage Kit (BioRad, France). Samples were diluted with lithium dodecyl sulphate buffer supplemented with reducing agents (Invitrogen) and then separated on 12\% NuPAGE Novex (Invitrogen). Proteins were transferred to nitrocellulose $(20 \mu \mathrm{g}$ for mouse lemurs, $10 \mu \mathrm{g}$ for control human brains), which were then saturated with $5 \%$ non-fat dried milk or $5 \%$ bovine serum albumin in TNT (Tris $15 \mathrm{mM} \mathrm{pH} 8, \mathrm{NaCl}$ $140 \mathrm{mM}, 0.05 \%$ Tween) and incubated at $4{ }^{\circ} \mathrm{C}$ for $24 \mathrm{~h}$ with the primary antibodies (TauCter (clone 9F6, LB labmade), TauNter (12-21, LB lab-made), Tau3R (Millipore ref 05,803), Tau4R (Millipore ref 05,804), TauE2 (Lot1, LB lab-made)). Appropriate HRP-conjugated secondary antibodies (anti- mouse PI-2000 and anti-rabbit PI-1000, Vector Laboratories) were incubated for $45 \mathrm{~min}$ at room temperature and signal was visualized using a chemoluminescence kit (ECL, Amersham Bioscience) and a LAS4000 imaging system (Fujifilm).

\section{Human brain samples}

Frozen brain samples (parietal cortex) from clinically different AD patients (four patients with classical slowly evolving forms of AD and four with a rapidly evolving form of $\mathrm{AD}$ ) and two age-matched control individuals were collected from a brain donation program of the GIE NeuroCEB and the National Reference Center (CNR)-prion brain banks. Consent forms were signed by either the patients themselves or their next of kin in their name, in accordance with French bioethics laws. The classical slowly evolving AD cases (AD1) were characterized by a disease duration of 5 to 8 years. The rapidly evolving $\mathrm{AD}$ cases (AD2) were characterized by a disease duration of 6 months to 3 years. No case of hippocampal sclerosis was reported and all brain samples (Ctrl, AD1, AD2) were $\operatorname{PrP}^{\mathrm{Sc}}$ negative. AD1 and AD2 brain samples were also negative for $\alpha$-synuclein and TDP-43. 

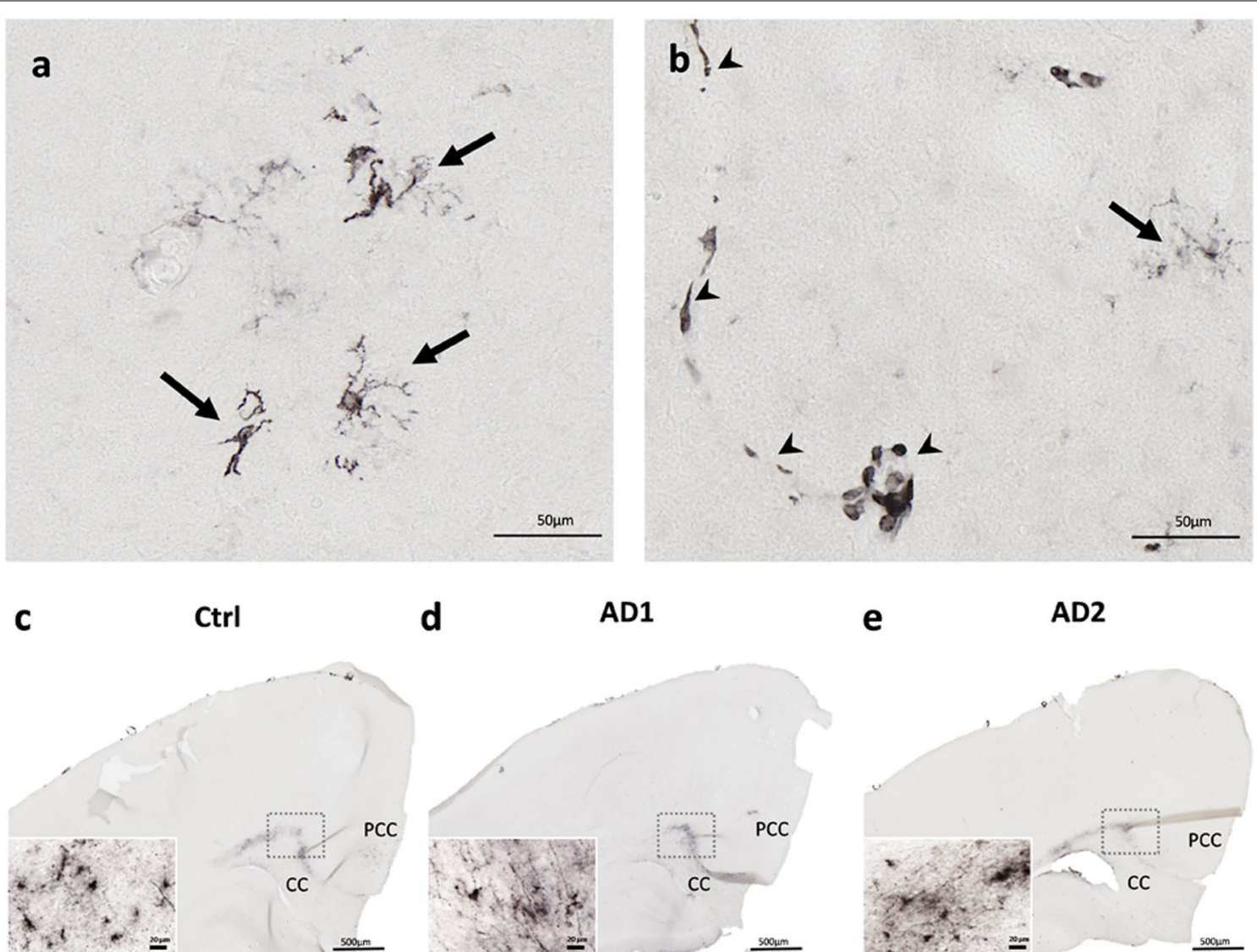

d

AD1

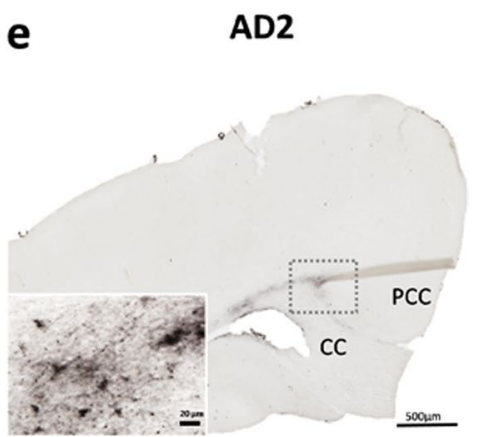

Fig. 10 Similar microglial response after the inoculation of Ctrl or AD brain extracts. Representative stainings of microgliosis (HLA-DR antibody) in the parenchyma (a-b, arrows) and in the vasculature (b, arrowheads). Staining was observed at the inoculation sites, i.e. the posterior cingulate cortex (PCC) and the corpus callosum (CC) (c-e) but not at distance of these regions. No difference was observed between the groups. Scale bars: $50 \mu \mathrm{m}$ in $\mathbf{a}-\mathbf{b}, 500 \mu \mathrm{m}$ in c-e and $20 \mu \mathrm{m}$ in inserts

\section{Neuropathological characterization}

All brain tissues were assessed by immunohistochemistry, as previously described in Gary et al. 2019 [11]. Briefly, 4- $\mu \mathrm{m}$-thick paraffin sections were cut, deparaffinized in xylene, successively rehydrated in ethanol (100, 90 , and $70 \%$ ) and rinsed under running tap water for $10 \mathrm{~min}$ before immunohistological staining. They were then incubated in $99 \%$ formic acid for $5 \mathrm{~min}$, quenched for endogenous peroxidase with $3 \%$ hydrogen peroxide and $20 \%$ methanol, and washed in water. Sections were blocked at room temperature for $30 \mathrm{~min}$ in $4 \%$ bovine serum albumin (BSA) in $0.05 \mathrm{M}$ tris-buffered saline, with $0.05 \%$ Tween 20, pH8 (TBS-Tween, Sigma). They were then incubated overnight at $+4{ }^{\circ} \mathrm{C}$ with the $6 \mathrm{~F} 3 \mathrm{D}$ anti-A $\beta$ antibody (Dako, $1 / 200$ ), polyclonal anti-tau antibody (Dako, 1/500), monoclonal anti-alpha-synuclein (LB509, Zymed, 1/250), polyclonal anti-TDP43 (Protein Tech Group, 1/1000) routinely used for $A \beta$, tau, alphasynuclein and TDP43 detection, respectively. Sections were further incubated with a biotinylated secondary antibody for $25 \mathrm{~min}$ at room temperature, and the presence of the secondary antibody was revealed by a streptavidin-horseradish peroxidase conjugate using diaminobenzidine (Dako, Glostrup, Denmark). Sliced were counterstained with Harris hematoxylin.

\section{Protein extraction for human brains}

For tau protein extraction, brain homogenates were sonicated on ice for $5 \mathrm{~min}$, centrifuged for $5 \mathrm{~min}$ at 3,000 $\times \mathrm{g}$ at $+4{ }^{\circ} \mathrm{C}$, diluted in $20 \mathrm{mM}$ Tris/2\% SDS and sonicated on ice for $5 \mathrm{~min}$. For $\mathrm{A} \beta$, Iba1 and GFAP protein extractions, brain homogenates were sonicated (6 strokes, cycle 0.5 , $30 \%$ amplitude) in a lysis buffer at a final concentration of $50 \mathrm{mM}$ Tris- $\mathrm{HCl} \mathrm{pH} 7.4,150 \mathrm{mM} \mathrm{NaCl}, 1 \%$ Triton$\mathrm{X}-100$ supplemented with $1 \mathrm{X}$ protease inhibitors (cOmplete $^{\mathrm{TM}}$ Mini, EDTA-free Protease Inhibitor Cocktail, Roche) and 1/100 diluted phosphatase inhibitors (Phosphatase Inhibitor Cocktail 2, Sigma-Aldrich). Samples were centrifuged at $20,000 \times \mathrm{g}$ for $20 \mathrm{~min}$ at $+4{ }^{\circ} \mathrm{C}$ and the supernatant was collected for further use. Extracted 

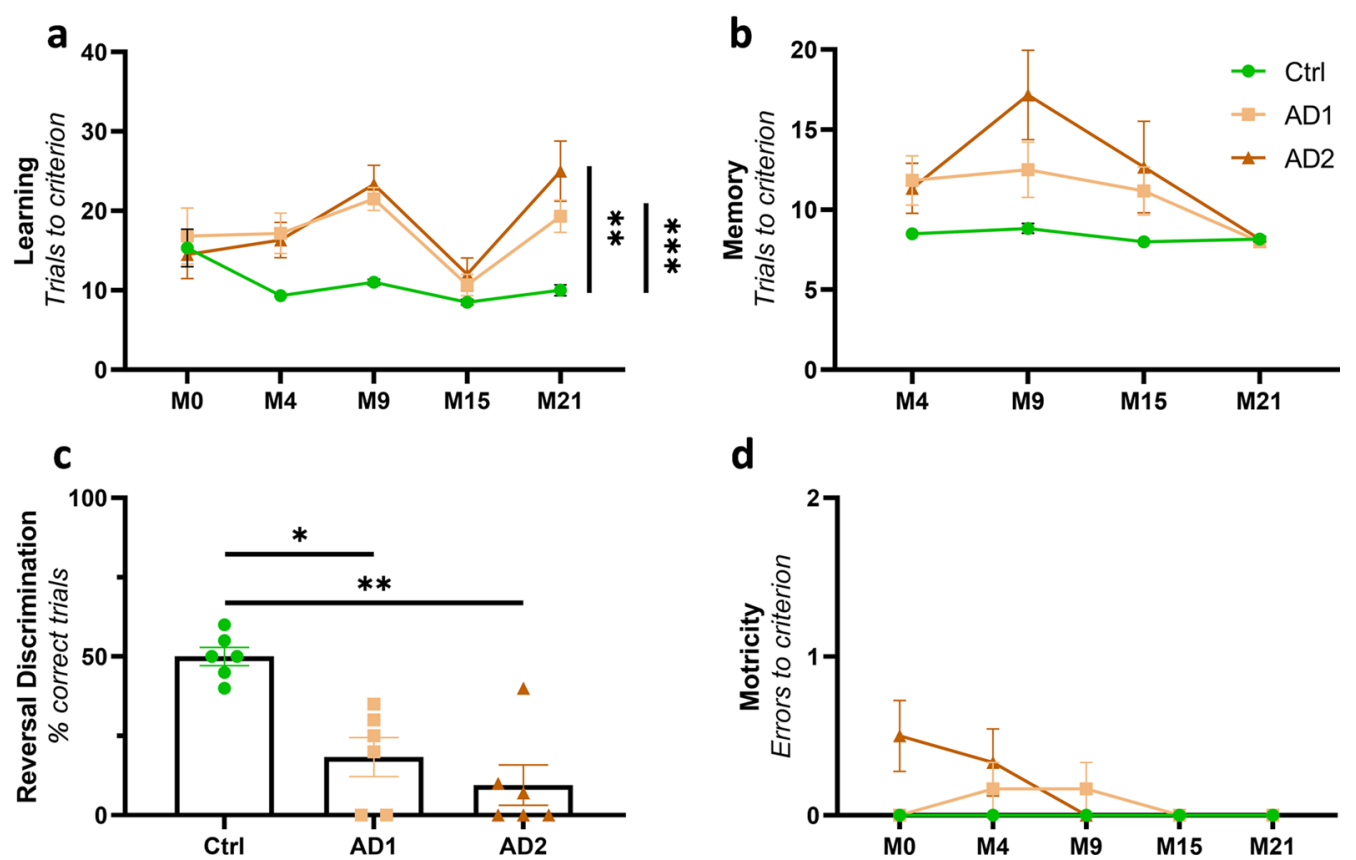

Fig. 11 Cognitive impairment induced by AD brain inoculations. a Longitudinal evaluation revealed learning deficits in AD groups compared to the Ctrl group (Time effect: $F_{(2.18,32.73)}=8.42, p=0.0009 ;$ Group effect: $F_{(2,15)}=9.52, p=0.002 ; p=0.0002$ and 0.004 , respectively for AD1 and AD2 compared to the Ctrl group; two-way ANOVA with the Geisser-Greenhouse correction, Tukey's multiple comparisons). b Memory performance comparison revealed a group effect throughout the follow-up, but no statistical difference between AD1, AD2 and Ctrl animals was detected following post-hoc evaluations (Time effect: $F_{(2.46,6.95)}=7.40, p=0.001$; Group effect: $F_{(2,15)}=3.83, p=0.045 ; p=0.066$ between AD2 and Ctrl at $9 \mathrm{mpi}, \mathrm{p}>0.17$ for every other comparisons; two-way ANOVA with the Geisser-Greenhouse correction, Tukey's multiple comparisons). c Reversal learning task performed at 21 mpi revealed deficits in AD groups compared with the Ctrl group ( $p=0.024$ and 0.0045, respectively for AD 1 and AD2; Kruskal-Wallis with Dunn's multiple comparisons). For all of the evaluated cognitive functions, no statistical difference was observed between AD1- and AD2-inoculated lemurs. $\mathbf{d}$ No motor impairment was observed with age and between the groups all throughout the follow-up (Time effect: $F_{(2.16,32.42)}=2.35, p=0.11$; Group effect: $F_{(2,15)}=2.57, p=0.11$; two-way ANOVA with the Geisser-Greenhouse correction, Tukey's multiple comparisons). ${ }^{*} p<0.05 ;{ }^{* *} p<0.01 ;{ }^{* * *} p<0.001$. Data are shown as mean \pm s.e.m

samples were stored at $-80{ }^{\circ} \mathrm{C}$ after evaluation of total protein concentration by a $\mathrm{BCA}$ assay $\left(\right.$ Pierce $\left.^{\mathrm{TM}}\right)$. The obtained concentration was used for the normalization of proteins. Total protein concentration was 5.40, 5.53 and $5.50 \mu \mathrm{g} / \mu \mathrm{l}$ respectively for the Ctrl, AD1 and AD2 homogenates.

\section{Western blots for human brain extracts}

For tau characterization, samples were diluted to $1 \mu \mathrm{g} /$ $\mu \mathrm{L}$, diluted in $2 \mathrm{X}$ lithium dodecyl sulfate (LDS, Thermo
Fisher Scientific) buffer with reducers and heated at $+100{ }^{\circ} \mathrm{C}$ for $10 \mathrm{~min} .15 \mu \mathrm{g}$ of samples were loaded on a $12 \%$ Bis-TrisCriterion $^{\text {TM }}$ gel (Bio-Rad) and migrated in MOPS buffer for $1 \mathrm{~h}$ at $165 \mathrm{~V}$ on ice. After protein transfer on nitrocellulose sheets, migration and quality of the transfer were checked with a ponceau S staining. The membrane was saturated for $1 \mathrm{~h}$ at room temperature, and was then incubated with the AT100 (pT212-pS214, Life technologies MN1060), 2H9 (pS422, 4BioDx 4BDX-1501), tau-Nter (12-21, LB lab-made) or

\section{(See figure on next page.)}

Fig. 12 Progressive cerebral atrophy induced by AD brain inoculation. Statistical heatmaps of t-values depicting regions in which grey matter volume decreased in AD-inoculated animals compared with Ctrl-inoculated ones (voxel-based morphometry, FDR-corrected $p<0.05$; voxel threshold extent $k=10$ ). To increase statistical power, AD1 and AD2-inoculated animals were grouped within a unique AD group. Longitudinal follow-up revealed a slight atrophy in the inoculated posterior cingulate region at $4 \mathrm{mpi}(\mathbf{a})$. No intergroup difference in brain volume was observed between 4 and $9 \mathrm{mpi}$ (b). Grey matter loss however extended to several other regions by $15 \mathrm{mpi}$ (c), without further progressing afterwards until $21 \mathrm{mpi}(\mathbf{d})$. Lateral $(\mathbf{e})$ and dorsal $(\mathbf{f})$ three-dimensional representations showing clusters of atrophied areas. The red arrow and crosses indicate the injection needle tract. 1-3: cerebral cortex areas 1-3, 4: cerebral cortex area 4, 5: cerebral cortex area 5, 6: cerebral cortex area 6, 7: cerebral cortex area 7, 17-18: cerebral cortex areas 17-18, AC: anterior cingulate cortex, BF: basal forebrain, Cd: caudate nucleus, Cl: claustrum, EC: entorhinal cortex, F: antero-medial frontal cortex, Fs: superior frontal cortex, H: hippocampus, Ins: insular cortex (areas 13-16), P: putamen, PA: peri-amygdalar cortex, PC: posterior cingulate cortex, RS: retrosplenial cortex, S: septum, Th: median thalamus [22] 

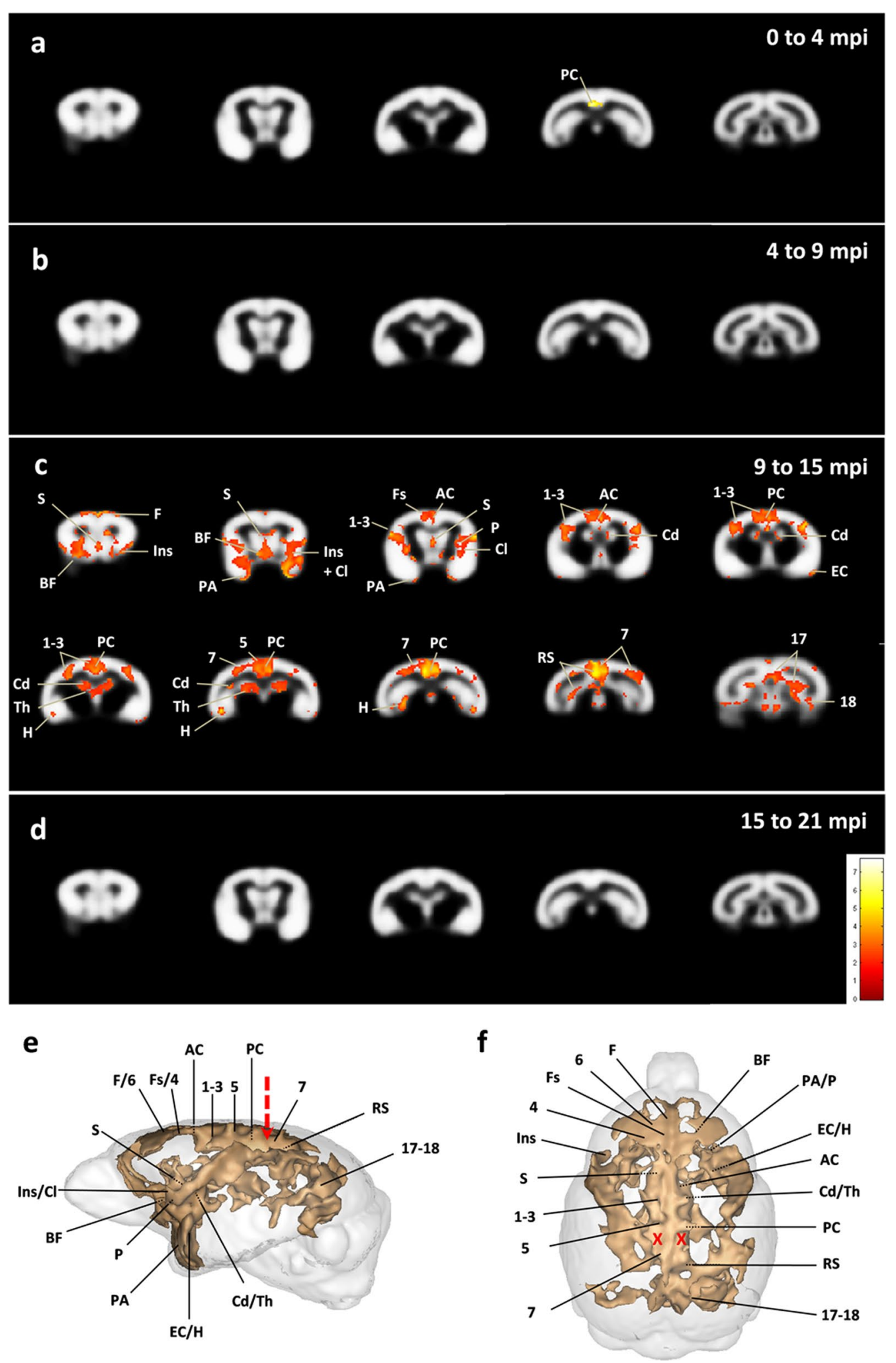

Fig. 12 (See legend on previous page.) 
tau-Cter (clone 9F6, LB lab-made) antibodies overnight at $+4{ }^{\circ} \mathrm{C}$. A peroxidase coupled secondary anti-rabbit or anti-mouse antibody was then applied for $45 \mathrm{~min}$ at room temperature. Immunoblotting was revealed by ECL. GAPDH (Sigma 9545) was used as a loading control. For Iba1 and GFAP evaluations, extracted samples were denatured at $+90{ }^{\circ} \mathrm{C}$ for $5 \mathrm{~min}$ in a buffer containing $1 \mathrm{X}$ LDS (NuPAGE ${ }^{\circledR}$ LDS sample buffer, Invitrogen) and DTT $1 \mathrm{X}\left(\mathrm{NuPAGE}^{\circledR}\right.$ sample reducing agent, Invitrogen). $10 \mu \mathrm{g}$ of denatured protein were loaded per well. Samples and molecular weight marker (Bio-Rad Precision Plus Protein ${ }^{\mathrm{TM}}$ Dual Color standards) were loaded on 4-20\% Criterion ${ }^{\mathrm{TM}}$ TGX $^{\mathrm{TM}}$ gels (Bio-Rad) and migration was performed in a $1 \mathrm{X}$ tris-glycine buffer (Bio$\mathrm{Rad})$ at $120 \mathrm{~V}$ for $1 \mathrm{~h}$. Proteins were then transferred to a nitrocellulose membrane using the Trans-Blot ${ }^{\circledR}$ Turbo $^{\text {TM }}$ (Biorad) system. Migration and quality of the transfer were checked with a ponceau $\mathrm{S}$ staining. The membrane was then blocked with a TBS/0.1\%Tween, 5\% milk solution for $1 \mathrm{~h}$ at RT, and incubated with the primary antibody Iba1 (Wako 1,919,741, 1/2000), GFAP (Dako Z0334, 1/5000) or actin (Sigma A2066, 1/5000) diluted in saturation buffer overnight at $+4{ }^{\circ} \mathrm{C}$. After washing in TBS/0.1\%Tween solution, the membrane was incubated with the appropriate secondary HRP-conjugate antibody diluted to $1 / 5000$ in TBS/0.1\% Tween for $1 \mathrm{~h}$ at RT. The chemiluminescent signal was revealed using the Clarity western ECL (Bio-Rad) kit and the Chemidoc ${ }^{\text {TM }}$ MP (Bio$\mathrm{Rad}$ ) imaging system. Protein band intensities were quantified on the ImageJ software and normalized by the actin expression level.

\section{ELISA quantifications}

For $A \beta$ protein quantification, all assay-specific material (pre-coated microtiter plate, buffers, antibodies, standard solutions) was provided in the V-PLEX kit A $\beta$ Peptide Panel 1 (6E10) $\left(\mathrm{MSD}^{\circledR}\right)$. Human brain homogenates were diluted to $1 / 5$ (Ctrl samples) or $1 / 10$ (AD1 and AD2 samples) in the dilution buffer. As described in the manufacturer's protocol, the microtiter plate was blocked for $1 \mathrm{~h}$ at RT with the appropriate buffer. After washing, $25 \mu$ l of detection antibody and $25 \mu$ l of diluted sample or standard were added in duplicate to the wells and incubated under continuous agitation for $2 \mathrm{~h}$ at RT. Wells were washed and $150 \mu \mathrm{l}$ of reading buffer was added. Plate reading was performed with the MSD Sector Imager 2400 (model 1200) multiplex assay system. $\mathrm{A} \beta_{1-38}, \mathrm{~A} \beta_{1-40}$ and $\mathrm{A} \beta_{1-42}$ quantifications were performed with the Discovery Workbench 4.0 MSD $^{\circledR}$ software. Tau protein quantifications (total tau and phosphot-tau181) were perfomed according to the manufacturer's protocol. Briefly, brain homogenates were diluted to $1 / 100$ and $1 / 200$ in the provided dilution buffer. $50 \mu$ l of standards or samples, as well as $50 \mu \mathrm{l}$ of detection antibody solution were added to wells and incubated for $14 \mathrm{~h}$ at $+4{ }^{\circ} \mathrm{C}$. After washing, $100 \mu \mathrm{l}$ of $1 \mathrm{X}$ anti-rabbit IgG HRP solution was added for a $30 \mathrm{~min}$ incubation period at RT. $100 \mu \mathrm{l}$ of stabilized chromogen were then added to each well for $30 \mathrm{~min}$ at RT, in the dark. The reaction was stopped by adding $100 \mu \mathrm{l}$ of Stop solution and the plate was read at $450 \mathrm{~nm}$ within the hour. Data were analyzed with GraphPad Prism 7 using the 4PL method. All samples were tested in duplicates.

\section{Human brain extracts preparation}

Parietal cortex samples were individually homogenized at $10 \%$ weight/volume $(\mathrm{w} / \mathrm{v})$ in a sterile $1 \mathrm{X}$ Dulbecco's phosphate buffer solution in CK14 soft tissue homogenizing tubes at $5000 \mathrm{rpm}$ for $20 \mathrm{~s}$ (Precellys ${ }^{\circledR}$, Bertin technologies). Brain extracts were then sonicated on ice for $5 \mathrm{~s}$ at $40 \%$ amplitude and centrifuged at $3000 \mathrm{~g}$ for $5 \mathrm{~min}$ at $+4{ }^{\circ} \mathrm{C}$. The supernatant was aliquoted in sterile polypropylene tubes and stored at $-80{ }^{\circ} \mathrm{C}$ until use.

Before the stereotaxic injection, $10 \% \mathrm{Ctrl}$, AD1 or AD2 individual brain extracts were thawed on ice and combined together according to their group. The three resulting combined samples (Ctrl, AD1 and AD2 brain extracts) were sonicated (70\% amplitude, 10 s on/off; Branson SFX 150 cell disruptor sonicator, $3.17 \mathrm{~mm}$ microtip probe Emerson, Bron) on ice in a sterile environment.

\section{Stereotaxic surgery}

Stereotaxic surgery was performed to infuse the brain extracts, bilaterally in the posterior cingulate cortex and the underlying corpus callosum. Mouse lemurs have a brain structure that can show significant interindividual variation. The stereotactic injection protocol developed during this study ensures that this variability is taken into account and therefore improves the accuracy of the injections. This protocol is based on a magnetic resonance imaging (MRI) acquisition followed by a surgical procedure. Mouse lemurs were maintained in a stable position on a non-magnetic bed, compatible with the MRI and stereotactic equipment, and for which the positions of the anesthetic mask, muzzle bar and ear bars were adjustable to fit the morphology of each animal. An intra-laboratory validation was performed prior to this study. Animals were fasted the day before the intervention. Water was available ad libitum up to $1 \mathrm{~h}$ before anesthesia.

Injection coordinates for the inoculated structures were calculated in reference to a landmark that crossed the middle of the ear bar for the antero-posterior axis, interhemispheric fissure for the left-right axis, and the skull surface for the dorso-ventral axis. We chose this landmark as it could be visualized both in MRI and under 
the surgeon's binocular magnifying glass. The coordinates of the targeted regions were defined on the MRI and were transposed into stereotaxic coordinates for the surgery.

The MR exams were performed using the following procedure. Mouse lemurs were pre-anesthetized by a subcutaneous injection of glycopyrronium bromide $\left(0.05 \mathrm{ml} / \mathrm{kg}\right.$; Robinul- $\left.\mathrm{V}^{\circledR}\right) 30 \mathrm{~min}$ before isoflurane anesthesia (induction at $4.5 \%$, maintenance at $1.5 \%$; Vetflurane $\left.{ }^{\circledR}\right)$. Throughout the procedure, their respiratory rhythm was monitored and body temperature was maintained at $37 \pm 0.5{ }^{\circ} \mathrm{C}$ using a heated blanket. The MRI system was an 11.7 Tesla Bruker BioSpec (Bruker, Ettlingen, Germany) running ParaVision 6.0.1. Images were acquired using a T2-weighted multi-slice multi-echo sequence with the following parameters: $\mathrm{TR}=7100 \mathrm{~ms}$; $\mathrm{TE}=24.20 \mathrm{~ms}$; echo spacing $=4.40 \mathrm{~ms}$; echo average $=10$; slice thickness $=0.23 \mathrm{~mm}$; number of slices $=128$; Axial-AP; Read/Phase/Slice: $\mathrm{Y} / \mathrm{X} / \mathrm{Z}$; resolution $=0.156 \times 0.156$ after zero-filling-interpolation $(2.5 \times 2.5)$; matrix $=104 \times 104$; field of view $=40 \times 40$ $\mathrm{mm}^{2}$; bandwidth $=100,000 \mathrm{~Hz}$; acquisition duration: $12 \min 18 \mathrm{~s}$.

Just after the MRI, animals were placed in a stereotactic frame (Phymep). Local anesthesia was performed with a subcutaneous injection of lidocaine $(5 \mathrm{mg} / \mathrm{kg}$; $0.5 \%$ Xylovet, Ceva), after cleaning the incision site with povidone iodine (Vétédine ${ }^{\circledR}$ ). Injections were performed bilaterally into the corpus callosum followed by the overlying posterior cingulate cortex, at the previously defined coordinates. Using $1 \mathrm{ml}$-Hamilton syringes and 34-gauge needles, $6.25 \mu \mathrm{l}$ of human brain homogenates were administered per injection site, at a $0.5 \mu \mathrm{l} / \mathrm{min}$ rate. At the end of each injection, needles were held in place for five additional minutes before removal. The incised area was cleaned with $10 \%$ povidone iodine (Vétédine ${ }^{\circledR}$ ) and sutured. Animals then received a subcutaneous injection of a $0.9 \%$ sodium chloride solution $(1 \mathrm{ml} / 100 \mathrm{~g})$ for rehydration, after which they were placed in a ventilated heating box $\left(25^{\circ} \mathrm{C}\right)$ and monitored until full recovery from anesthesia. Two weeks after the injection, an MRI was performed to evaluate potential post-operative complications (increased inflammation, cerebral hemorrhage). No imaging abnormalities were detected in any animals.

\section{Behavioral tests}

Learning, long term memory performance and reversal learning abilities were evaluated using discrimination tests in a jumping stand apparatus [31]. Motor skills were evaluated using a tower test [25]. All tests were conducted using devices adapted to lemurs developed by our team or other mouse lemur specialists.
Visual discrimination test in a jumping stand apparatus The jumping stand apparatus evaluates lemurs' learning and long-term memory performances using discrimination tests [11, 31] (Additional File 2). The device is a vertical cage made of plywood walls, except for the front panel which consists in a one-way mirror allowing observation. During the test, the animal is placed on a elevated central platform and must jump from this starting platform to one of the two landing platforms. If no spontaneous jump is performed within a minute, the central platform can be tilted slightly downwards, creating a slippery slope, to encourage the animal to jump. Each landing platform is associated with a different visual stimulus, characterized by a specific shape, texture and pattern. For each pair of visual stimuli, one is associated with a positive reinforcement (i.e. a stable platform giving access to a 2-min rest in a wooden nesting box), whereas the other one is associated with a negative reinforcement (i.e. an unstable platform leading to a fall to the bottom of the cage). After a fall, the mouse lemur is left at the bottom of the cage for $20 \mathrm{~s}$ before the next trial. As mouse lemurs prefer confined spaces, reaching their nesting box when placed in an open space is a strong motivator for behavioral testing. During a discrimination task, the mouse lemur had to identify the positive stimulus, the location of which was randomly alternated between the two landing platforms over the course of the test and changed at least every three trials. The test ended when the success criterion (i.e. at least 8 correct choices out of 10 consecutive attempts) was reached. The score given to an animal during learning and memory tasks was the number of trials required to reach this criterion.

Before the first test, an habituation session consisting of 7 trials was carried out. During the first four trials, only one stable central landing platform was available in front of the nesting box opening. Trial 1 was characterized by the presence of a board directly connecting the departure platform to the landing platform, so that jumping was not required to access the nesting box. This board was removed for trial 2, during which the lemur needed to jump to reach the stable landing platform. During trial 3 and 4, an opaque screen was placed above the landing platform in order to hide the opening of the nesting box. The animal therefore needed to jump onto the stable landing platform and go under the screen to reach the reward. During trial 5 to 7 , an unstable platform was introduced, and alternately placed either on the right or on left of the nesting box entrance.

Learning abilities were evaluated as the lemur had to identify the positive stimulus out of the two stimuli to reach its nesting box. Long-term memory performance reflected the ability of mouse lemurs to remember the positive stimulus presented 4 or 6 months earlier. In 
order to create experimental groups with comparable learning capacities, the learning test was carried out before the inoculation with human brain homogenates (M0). Memory performance was evaluated 4 months later, followed (one day later) by a new learning task. Longitudinal evaluation was performed by a succession of learning and memory tasks at 4, 9, 15 and 21 months post-inoculation. At each timepoint, a new pair of visual stimuli was introduced to the animals for the new learning task. The third cognitive task was a reversal learning task conducted at $21 \mathrm{mpi}$. After a successful discrimination learning session, the outcomes associated with the two stimuli were reversed. Twenty consecutive trials were performed for each animal and the percentage of correct choices was compared between the groups.

Motricity test The tower test was designed to evaluate motor performances of the lemurs through the achievement of successive high jumps [25]. Similarly to the jumping stand apparatus, it is based on the strong motivation of mouse lemurs to reach their nesting box in order to escape the discomfort felt in open spaces. The device is a $180 \times 35 \times 35 \mathrm{~cm}$ tower with three opaque sides and one transparent Plexiglas side. It is crossed horizontally by seven metal rods with a diameter of $5 \mathrm{~mm}$ and gradually spaced from one another by $10 \mathrm{~cm}$ (at the bottom) to $30 \mathrm{~cm}$ (at the top). Jumps with increasing difficulty need to be completed in order to reach the reward. A nesting box can be positioned at three different levels, making it accessible after reaching rod 5 (low position), 6 (intermediate position) or 7 (high position). The test was monitored by a camera placed in front of the tower.

During the test, the lemur was placed on the floor of the tower and had to jump from rod to rod in order to reach the nesting box made accessible after jumping on rod 7. Jumps were either performed spontaneously or after a stimulus in case of immobility ( $>3 \mathrm{~min}$ ). This stimulus could be visual (introduction of a visual stimulus at the bottom of the tower), or mechanical in case of a more prolonged inactivity when on a rod (rotation of the rod on which the lemur was located). When the animal reached the nesting box, it was rewarded with a 5-min rest inside.

When the animal was introduced to the device for the first time, an habituation session consisting of six trials was carried out. Throughout the trials, the nesting box was gradually moved upwards, from the lower position during trial 1 and 2, to the intermediate position during trial 3 and 4, and finally to the upper position during trial 5 and 6.

The motricity test phase consisted of three consecutive trials during which the nesting box was placed at the upper position. The number of falls between the last two rods, spaced by $30 \mathrm{~cm}$, was used as a criterion reflecting motor performances. Motricity tests were performed following the visual discrimination test before humain brain inoculation and at 4, 9, 15 and 21 months post-inoculation.

\section{Morphological MRI}

Brain volume evolution was evaluated throughout the study, starting before inoculation and then at 4, 9, 15 and 21 months post-inoculation. All images were acquired on a 11.7 Tesla MRI (Bruker Corporation), using the following parameters: $\mathrm{TR}=8000 \mathrm{~ms}$; $\mathrm{TE}=28.05 \mathrm{~ms}$; echo spacing $=5.10 \mathrm{~ms}$; echo average $=10$; slice thickness $=0.23 \mathrm{~mm}$; number of slices $=128$, Axial-AP; Read/Phase/Slice: $\mathrm{Y} / \mathrm{X} / \mathrm{Z} ; \quad$ resolution $=0.115 \times 0.115$ after zero-filling interpolation $(1.34 \times 1.34)$; matrix $=192 \times 192$; field of view $=29.44 \times 29.44 \mathrm{~mm}^{2}$; bandwidth $=100,000 \mathrm{~Hz}$; acquisition duration: $25 \mathrm{~min} 36 \mathrm{~s}$. Animals were anesthetized and monitored as previously described for stereotaxic injections.

Images were analyzed using voxel-based morphometry by applying SPM8 (Wellcome Trust Institute of Neurology, University College London, UK, www.fil.ion.ucl.ac. $\mathrm{uk} / \mathrm{spm}$ ) with the SPMMouse toolbox (http://spmmo use.org) for animal brain morphometry using a procedure already implemented for mouse lemurs [11, 36]. The brain images were segmented into grey (GM) and white matter (WM) tissue probability maps using locally developed priors, then spatially transformed to the standard space, defined by Sawiak et al., using a GM mouse lemur template [36]. Affine regularization was set for an average-sized template, with a bias non-uniformity FWHM cut-off of $10 \mathrm{~mm}$ and a $5 \mathrm{~mm}$ basis-function cut off and sampling distance of $0.3 \mathrm{~mm}$. The resulting GM and WM portions were output in rigid template space, and DARTEL [1] was used to create non-linearly registered maps for each subject and common templates for the cohort of animals. The warped GM portions for each subject were adjusted using the Jacobian determinant from the DARTEL registration fields to preserve tissue amounts ("optimized VBM" [13]) and smoothed with a Gaussian kernel of $600 \mu \mathrm{m}$ to produce maps for analysis.

A general linear model was designed to evaluate relative changes in GM values as a function of time between the control- and Alzheimer's disease-inoculated groups. This type of analysis produces t-statistics and colorcoded maps that are the product of a statistical analysis performed at every voxel in the brain. Contiguous groups of voxels that attain statistical significance, called clusters, are displayed on brain images. To control for multiple comparisons, an adjusted p-value was calculated using the voxel-wise false discovery rate (FDR-corrected $\mathrm{p}<0.05)$, with extent threshold values of 10 voxels, 
meaning that clusters required 10 contiguous voxels to be selected as relevant [12]. Voxels with a modulated GM value below 0.2 were not considered for statistical analysis. The operator was blinded to the group attribution during image processing.

\section{Histology}

Mouse lemurs were sacrificed at $21 \mathrm{mpi}$ with an intraperitoneal injection of pentobarbital sodium $(0.1 \mathrm{ml} / 100 \mathrm{~g}$; Exagon, Axience). Twenty minutes before sacrifice, a subcutaneous administration of buprenorphine $\left(0.1 \mathrm{mg} / 100 \mathrm{~g}\right.$; Vétergésic $\left.{ }^{\circledR}\right)$ was performed for analgesia. Animals were perfused intracardiacally with cold sterile $0.1 \mathrm{M}$ PBS for $4 \mathrm{~min}$, at a rate of $8 \mathrm{ml} / \mathrm{min}$. The brain was post-fixed in $4 \%$ paraformaldehyde for $48 \mathrm{~h}$ at $+4{ }^{\circ} \mathrm{C}$, transferred in a $15 \%$ sucrose solution for $24 \mathrm{~h}$ and in a $30 \%$ sucrose solution for $48 \mathrm{~h}$ at $+4{ }^{\circ} \mathrm{C}$ for cryoprotection. Serial coronal sections of $40 \mu \mathrm{m}$ were performed with a microtome (SM2400, Leica Microsystem) and stored at $-20{ }^{\circ} \mathrm{C}$ in a storing solution (glycerol $30 \%$, ethylene glycol $30 \%$, distilled water $30 \%$, phosphate buffer $10 \%)$. Free-floating sections were rinsed in a $0.1 \mathrm{M}$ PBS solution (10\% Sigma-Aldrich ${ }^{\circledR}$ phosphate buffer, $0.9 \%$ Sigma-Aldrich ${ }^{\circledR} \mathrm{NaCl}$, distilled water) before use.

Immunohistology, Thioflavin S and Gallyas silver staining Mouse lemur brain sections were stained for amyloid- $\beta$ (4G8 and A $\beta 42$ antibodies, Thioflavin S), tau (AT8, AT100, pS422 antibodies and Gallyas silver staining) and microglia (HLA-DR antibody). A pretreatment with $70 \%$ formic acid $\left(\mathrm{VWR}^{\circledR}\right)$ for 20 min at room temperature (RT) was performed for the 4 G8 labelling. For the HLA-DR and AT100 stainings, the pretreatment consisted in a 30 min-long incubation in EDTA $1 \mathrm{X}$ citrate (Diagnostic BioSystems ${ }^{\circledR}$ ) at $95{ }^{\circ} \mathrm{C}$ followed by rinsings in $0.5 \%$ Triton X-100/0.05 M Tris-HCl Buffered Saline solution (TBS) or a PBS solution respectively, for $2 \times 10 \mathrm{~min}$. For the 4G8, A $\beta 42$, AT8, AT100, pS422 and HLA-DR stainings, tissues were then incubated in hydrogen peroxide $\mathrm{H}_{2} \mathrm{O}_{2} 30 \%$ (Sigma-Aldrich ${ }^{\circledR}$ ) diluted $1 / 100$ for $20 \mathrm{~min}$ to inhibit endogenous peroxidases. Blocking of non-specific antigenic sites was achieved over $1 \mathrm{~h}$ using a $0.2 \%$ Triton X-100/0.1 M PBS (Sigma-Aldrich ${ }^{\circledR}$ ) (PBST) or $0.5 \%$ Triton $\mathrm{X}-100 / 0.05 \mathrm{M}$ TBS (TBST) solution containing $3 \%$ bovine serum albumin or $4.5 \%$ normal goat serum, depending on the staining. Sections were then incubated at $+4{ }^{\circ} \mathrm{C}$ with the 4G8 (Biolegend 800,706, 1/350), A $\beta 42$ (Invitrogen $44,344,1 / 500$ ) or pS422 (Abcam 79,415, 1/1000) antibody diluted in a $3 \%$ BSA/PBST solution for $48 \mathrm{~h}$ or for $96 \mathrm{~h}$ with the AT8 antibody (Thermo MN1020B, 1/500), in a 3\%BSA/ TBST solution with the HLA-DR antibody (Dako M0746) for $48 \mathrm{~h}$ or in a $5 \% \mathrm{NGS} / \mathrm{TBST}$ solution with the AT100 antibody (Thermo MN1060, 1/250) for $96 \mathrm{~h}$. After rinsing, an incubation with the appropriate biotinylated secondary antibody diluted to $1 / 1000$ in PBST or TBST was performed for $1 \mathrm{~h}$ at RT, followed by a $1 \mathrm{~h}$ incubation at RT with a 1:250 dilution of an avidin-biotin complex solution $\left(A B C\right.$ Vectastain kit, Vector Laboratories $\left.{ }^{\circledR}\right)$. Revelation was performed using the DAB Peroxidase Substrate Kit (DAB SK4100 kit, Vector Laboratories ${ }^{\circledR}$ ). Sections were mounted on Superfrost Plus slides (Thermo-Scientific ${ }^{\circledR}$ ). For the 4G8, A $\beta 42$, AT8, AT100 and pS422 labellings, a cresyl violet counterstain was performed. All sections were then dehydrated in successive baths of ethanol at $50^{\circ}$, $70^{\circ}, 96^{\circ}$ and $100^{\circ}$ and in xylene. Slides were mounted with the Eukitt ${ }^{\circledR}$ mounting medium $\left(\mathrm{Chem}-\mathrm{Lab}^{\circledR}\right)$.

For the Thioflavin $\mathrm{S}$ staining, free-floating sections were first mounted on Superfrost Plus (Thermo-Scientific $\left.^{(}\right)$slides and dried overnight. Sections were then incubated with a Thioflavin S (Sigma T1892) solution for $20 \mathrm{~min}$ at RT. They were then incubated in ethanol $100^{\circ}$ and mounted with the FluorSave medium.

Free-floating sections were mounted on Superfrost Plus (Thermo-Scientific ${ }^{\circledR}$ ) slides and dried overnight prior to Gallyas staining. All steps of the Gallyas staining were performed between 20 and $25^{\circ} \mathrm{C}$. Section were permeabilized by successive incubations in toluene $(2 \times 5 \mathrm{~min})$ followed by ethanol at $100^{\circ}, 90^{\circ}$ and $70^{\circ}$ ( 2 min per solution). Slides were then incubated in a $0.25 \%$ potassium permanganate solution for $15 \mathrm{~min}$, in $2 \%$ oxalic acid for $2 \mathrm{~min}$ then in a lanthanum nitrate solution $(0.04 \mathrm{~g} / \mathrm{l}$ lanthanum nitrate, $0.2 \mathrm{~g} / \mathrm{l}$ sodium acetate, $10 \% \mathrm{H} 2 \mathrm{O} 230 \%$ ) for $1 \mathrm{~h}$ to reduce non-specific background. Several rinses with distilled water were performed followed by an incubation in an alkaline silver iodide solution (3.5\% AgNO3 $1 \%, 40 \mathrm{~g} / \mathrm{l} \mathrm{NaOH}, 100 \mathrm{~g} / \mathrm{l} \mathrm{KI}$ ) for $2 \mathrm{~min}$. The reaction was neutralized with $0.5 \%$ glacial acetic acid baths $(3 \times 1 \mathrm{~min})$ and sections were incubated for $20 \mathrm{~min}$ in a developing solution (2 g/l NH4NO3, 2 g/l AgNO3, 10 g/l tungstosilicilic acid, $0.76 \%$ formaldehyde $37 \%, 50 \mathrm{~g} / \mathrm{l}$ anhydrous $\mathrm{Na} 2 \mathrm{CO} 3)$. Several rinses with $0.5 \%$ acetic acid $(3 \times 1 \mathrm{~min})$ followed by an incubation in $1 \%$ gold chloride solution for $5 \mathrm{~min}$ were then carried out. Sections were rinsed with distilled water and the staining was fixed with a $1 \%$ sodium thiosulfate solution. All sections were then rinsed with distilled water and dehydrated for 1 to $5 \mathrm{~min}$ in successive baths of ethanol at $50^{\circ}, 70^{\circ}, 96^{\circ}$ and $100^{\circ}$ and in xylene. Slides were mounted with Eukitt ${ }^{\circledR}$ mounting medium $\left(\mathrm{Chem}-\mathrm{Lab}^{\circledR}\right)$.

\section{Image analysis}

Z-stack images were acquired at $20 \times$ (z-stacks with 16 planes, $1 \mu \mathrm{m}$ steps with extended depth of focus) using an Axio Scan.Z1 (Zeiss ${ }^{\circledR}$ ). Each slice was extracted individually in the.czi format using the Zen 2.0 (Zeiss ${ }^{\circledR}$ ) software. Image processing and analysis were performed with the 
ImageJ software (https://imagej.nih.gov/ij/). Images were imported with a $50 \%$ reduction in resolution $(0.44 \mu \mathrm{m} /$ pixel), converted to the RGB format and compressed in.tif format.

For the 4G8 immunostaining, the blue component of each image was extracted along with the background in order to remove the cresyl violet counter-staining from the analysis. Segmentation was performed with a manual threshold set at 202/255. Clusters of pixels with a radius below $15 \mu \mathrm{m}$ were removed. A $\beta$ load was evaluated as a percentage of 4G8-positive surface area in each ROI, except for the hippocampus. In the hippocampus, the number of plaques was manually counted as $\mathrm{A} \beta$ deposition was mainly concentrated in some areas and the evaluation of an overall staining was not appropriate. For the AT8 staining, AD-like neurofibrillary tangles quantification was performed by manually counting the lesions and neuropil threads were evaluated by using a semi-quantitative scoring system based on the intensity and extent of the lesions $(0=$ absent, $1=$ slight to moderate, $2=$ moderate to severe). For the HLA-DR staining, a semi-quantitative analysis was performed by assigning a severity score based on the intensity and extent of the staining at the inoculation sites $(0=$ absent, $1=$ slight, $2=$ moderate, $3=$ severe). All quantifications were performed on adjacent slices between $\mathrm{A} 4.50 \mathrm{~mm}$ and $\mathrm{P} 1.50 \mathrm{~mm}$ from bregma. 4G8 and AT8 were analyzed on 10 slices, HLADR on 3 slices adjacent to the inoculation site. ROIs were manually segmented using a mouse lemur atlas previously presented by our group [27], which was based on the reference atlases by Le Gros Clark [22] and Bons [4].

The three-dimensional rendering of $A \beta$ and tau pathologies in mouse lemur brains was based on regions of interests defined in a digital atlas of mouse lemurs [27]. $A \beta$ or tau lesions were reported within regions of interests using a three-level semi-quantitative scale (no lesions/intermediate/high lesion load). The ITK-SNAP software (http://www.itksnap.org) was used to create the surface/2D renderings of lesion loads.

\section{Statistical analysis}

Statistical analysis was performed using the GraphPad Prism software 9. For human brain characterization, data are shown as mean \pm standard deviation of the replicates. Mouse lemur histological data are shown on scattered dot plots with mean \pm standard error of the mean (s.e.m.) and Mann-Whitney's test was carried out to compare results between the AD1 and AD2 groups. Behavioral studies data are shown as mean \pm standard error of the mean (s.e.m) to represent performance evolution over time. Cognitive and motor performances which included repeated measures were evaluated using a two-way repeated measures
ANOVA with the Geisser-Greenhouse correction and Tukey's multiple comparisons. The significance level was set at $p<0.05$.

\section{Supplementary Information}

The online version contains supplementary material available at https://doi. org/10.1186/s40478-021-01266-8.

Additional file 1. Supplementary table and figures.

Additional file 2. Movie showing three consecutive trials in a jumping stand apparatus.

\section{Acknowledgements}

The project was funded by the Association France-Alzheimer-2016. S.L. was founded by the Ministère de l'Enseignement Supérieur, de la Recherche et de l'Innovation, and by the Fondation pour la Recherche Médicale-2020. The project was performed in a core facility supported by/member of NeurATRIS-ANR-11-INBS-0011. It was also supported by internal funds from the Laboratory of Neurodegenerative Diseases UMR9199 CEA/CNRS and MIRCen. We thank the donors and the Brain Donation Program of the "The Brainbank Neuro-CEB Neuropathology Network" run by a consortium of Patient Associations: ARSLA (association for research on amyotrophic lateral sclerosis), CSC (cerebellar ataxias), Fondation ARSEP (association for research on multiple sclerosis), France DFT (fronto-temporal dementia), Fondation Vaincre Alzheimer, France Parkinson, with the support of Fondation Plan Alzheimer and IHU A-ICM for providing the brain samples used in this study. We thank James Koch (University of Wisconsin Oshkosh, USA) for critical review of this article.

\section{Authors' contributions}

S.L., F.P., A.S.H., J.L.P., and M.D. contributed to the study conception and design. N.N.N., S.H., C.D. provided the human brain samples. N.N.N., S.L., S.B., C.D. and S.H. characterized the human brain samples. S.L. and M.G. performed the inoculations in mouse lemurs. J.L.P. designed and performed cognitive evaluations. F.P., S.L., A.S.H. designed and performed the immunohistological analysis in animals. S.E., L.B., A.S.H., and S.L. performed biochemical analysis. S.L., J.L.P. and M.D. wrote the manuscript. All authors commented on previous versions of the manuscript. All authors read and approved the final manuscript.

\section{Declarations}

\section{Competing interests}

The authors declare no competing financial interests.

\section{Author details}

${ }^{1}$ Laboratoire des Maladies Neurodégénératives, Université Paris-Saclay, CEA, CNRS, 18 Route du Panorama, 92265 Fontenay-aux-Roses, France. ${ }^{2}$ Commissariat à l'Energie Atomique et aux Energies Alternatives (CEA), Direction de la Recherche Fondamentale (DRF), Institut François Jacob, MIRCen, 18 Route du Panorama, 92265 Fontenay-aux-Roses, France. ${ }^{3}$ Institut du Cerveau, UMR 7225, Sorbonne Université, Paris Brain Institute-ICM, CNRS, AP-HP, Hôpital de la Pitié Salpêtrière, Inserm U1127 DMU Neurosciences, Paris, France. ${ }^{4}$ Brainbank NeuroCEB Neuropathology Network: Plateforme de Ressources Biologiques, Hôpital de La Pitié-Salpêtrière, Bâtiment Roger Baillet, 47-83 boulevard de I'Hôpital, 75651 Paris Cedex 13, France. ${ }^{5}$ Université de Lille, CHU-Lille, LabEx DISTALZ, Alzheimer \& Tauopathies, Rue Polonovski, 59045 Lille, France. ${ }^{6}$ Laboratory of Cognitive Functioning and Dysfunctioning (DysCo), University of Paris 8, 93526 cedex Saint-Denis, France. ${ }^{7}$ Angers, France. ${ }^{8}$ Bordeaux, France. ${ }^{9}$ Caen, France. ${ }^{10}$ Clermont-Ferrand, France. ${ }^{11}$ Grenoble, France. ${ }^{12}$ Lille, France.

${ }^{13}$ Limoges, France. ${ }^{14}$ Lyon, France. ${ }^{15}$ Marseille, France. ${ }^{16}$ Montpellier, France.

${ }^{17}$ Nice, France. ${ }^{18}$ Paris, France. ${ }^{19}$ Rennes, France. ${ }^{20}$ Rouen, France. ${ }^{21}$ Strasbourg, France.

Received: 28 July 2021 Accepted: 22 September 2021

Published online: 12 October 2021 


\section{References}

1. Ashburner J (2007) A fast diffeomorphic image registration algorithm. Neuroimage 38:95-113. https://doi.org/10.1016/j.neuroimage.2007.07. 007

2. Baker HF, Ridley RM, Duchen LW, Crow TJ, Bruton CJ (1993) Evidence for the experimental transmission of cerebral beta-amyloidosis to primates. Int J Exp Pathol 74:441-454

3. Baron JC, Chetelat G, Desgranges B, Perchey G, Landeau B, de la Sayette $V$, Eustache $F(2001)$ In vivo mapping of gray matter loss with voxel-based morphometry in mild Alzheimer's disease. Neuroimage 14:298-309

4. Bons N, Sihol S, Barbier V, Mestre-Frances N, Albe-Fessard D (1998) A stereotaxic atlas of the grey lesser mouse lemur brain (Microcebus murinus). Brain Res Bull 46:1-173. https://doi.org/10.1016/S0361-9230(97) 00458-9

5. Buckner RL, Sepulcre J, Talukdar T, Krienen FM, Liu H, Hedden T, AndrewsHanna JR, Sperling RA, Johnson KA (2009) Cortical hubs revealed by intrinsic functional connectivity: mapping, assessment of stability, and relation to Alzheimer's disease. J Neurosci 29:1860-1873. https://doi.org/ 10.1523/JNEUROSCI.5062-08.2009

6. Clavaguera F, Akatsu H, Fraser G, Crowther RA, Frank S, Hench J, Probst A, Winkler DT, Reichwald J, Staufenbiel M, Ghetti B, Goedert M, Tolnay M (2013) Brain homogenates from human tauopathies induce tau inclusions in mouse brain. Proc Natl Acad Sci USA 110:9535-9540. https://doi. org/10.1073/pnas.1301175110

7. Clavaguera F, Bolmont T, Crowther RA, Abramowski D, Frank S, Probst A, Fraser G, Stalder AK, Beibel M, Staufenbiel M, Jucker M, Goedert M, Tolnay M (2009) Transmission and spreading of tauopathy in transgenic mouse brain. Nat Cell Biol 11:909-913

8. Dhenain M (2001) Age-related cognitive and neurobiological alterations in animals. In: Boller F, Cappa S (eds) Handbook of neuropsychology, 2nd edn. Elsevier, Amsterdam, pp 1-21

9. Duyckaerts C, Sazdovitch V, Ando K, Seilhean D, Privat N, Yilmaz Z, Peckeu L, Amar E, Comoy E, Maceski A, Lehmann S, Brion JP, Brandel JP, Haik S (2018) Neuropathology of iatrogenic Creutzfeldt-Jakob disease and immunoassay of French cadaver-sourced growth hormone batches suggest possible transmission of tauopathy and long incubation periods for the transmission of Abeta pathology. Acta Neuropathol 135:201-212. https://doi.org/10.1007/s00401-017-1791-x

10. Garin CM, Nadkarni NA, Landeau B, Chételat G, Picq J-L, Bougacha S, Dhenain M (2021) Resting state functional atlas and cerebral networks in mouse lemur primates at 117 Tesla. Neurolmage 226:117589. https://doi. org/10.1016/j.neuroimage.2020.117589

11. Gary C, Lam S, Herard AS, Koch JE, Petit F, Gipchtein P, Sawiak SJ, Caillierez R, Eddarkaoui S, Colin M, Aujard F, Deslys JP, Network FN, Brouillet E, Buée L, Comoy EE, Pifferi F, Picq J-L, Dhenain M (2019) Encephalopathy induced by Alzheimer brain inoculation in a non-human primate. Acta Neuropathol Commun. https://doi.org/10.1186/s40478-019-0771-x

12. Genovese CR, Lazar NA, Nichols T (2002) Thresholding of statistical maps in functional neuroimaging using the false discovery rate. Neuroimage 15:870-878. https://doi.org/10.1006/nimg.2001.1037

13. Good CD, Johnsrude IS, Ashburner J, Henson RN, Friston KJ, Frackowiak RS (2001) A voxel-based morphometric study of ageing in 465 normal adult human brains. Neuroimage 14:21-36. https://doi.org/10.1006/ nimg.2001.0786

14. Herve D, Porche M, Cabrejo L, Guidoux C, Tournier-Lasserve E, Nicolas G, Adle-Biassette H, Plu I, Chabriat H, Duyckaerts C (2018) Fatal Abeta cerebral amyloid angiopathy 4 decades after a dural graft at the age of 2 years. Acta Neuropathol 135:801-803. https://doi.org/10.1007/ s00401-018-1828-9

15. Hong M, Zhukareva V, Vogelsberg-Ragaglia V, Wszolek Z, Reed L, Miller Bl, Geschwind DH, Bird TD, McKeel D, Goate A, Morris JC, Wilhelmsen KC, Schellenberg GD, Trojanowski JQ, Lee VMY (1998) Mutation-specific functional impairments in distinct Tau isoforms of hereditary FTDP-17. Science 282:1914-1917. https://doi.org/10.1126/science.282.5395.1914

16. Jaunmuktane Z, Banerjee G, Paine S, Parry-Jones A, Rudge P, Grieve J, Toma AK, Farmer SF, Mead S, Houlden H, Werring DJ, Brandner S (2021) Alzheimer's disease neuropathological change three decades after iatrogenic amyloid-beta transmission. Acta Neuropathol 142:211-215. https:// doi.org/10.1007/s00401-021-02326-y

17. Jaunmuktane Z, Mead S, Ellis M, Wadsworth JD, Nicoll AJ, Kenny J, Launchbury F, Linehan J, Richard-Loendt A, Walker AS, Rudge P, Collinge
J, Brandner S (2015) Evidence for human transmission of amyloid-beta pathology and cerebral amyloid angiopathy. Nature 525:247-250. https:// doi.org/10.1038/nature15369

18. Jucker M, Walker LC (2018) Propagation and spread of pathogenic protein assemblies in neurodegenerative diseases. Nat Neurosci 21:1341-1349. https://doi.org/10.1038/s41593-018-0238-6

19. Kunishio K, Haber SN (1994) Primate cingulostriatal projection - limbic striatal versus sensorimotor striatal input. J Comp Neurol 350:337-356. https://doi.org/10.1002/cne.903500302

20. Langer F, Eisele YS, Fritschi SK, Staufenbiel M, Walker LC, Jucker M (2011) Soluble Abeta seeds are potent inducers of cerebral beta-amyloid deposition. J Neurosci 31:14488-14495. https://doi.org/10.1523/JNEUROSCl. 3088-11.2011

21. Lauwers E, Lalli G, Brandner S, Collinge J, Compernolle V, Duyckaerts C, Edgren G, Haik S, Hardy J, Helmy A, Ivinson AJ, Jaunmuktane Z, Jucker M, Knight R, Lemmens R, Lin IC, Love S, Mead S, Perry VH, Pickett J, Poppy G, Radford SE, Rousseau F, Routledge C, Schiavo G, Schymkowitz J, Selkoe DJ, Smith C, Thal DR, Theys T, Tiberghien P, van den Burg P, Vandekerckhove P, Walton C, Zaaijer HL, Zetterberg H, De Strooper B (2020) Potential human transmission of amyloid beta pathology: surveillance and risks. Lancet Neurol 19:872-878. https://doi.org/10.1016/S1474-4422(20) 30238-6

22. Le Gros Clark WE (1931) The brain of Microcebus murinus. Proc Zool Soc London 101:463-486

23. Maclean CJ, Baker HF, Ridley RM, Mori H (2000) Naturally occurring and experimentally induced beta-amyloid deposits in the brains of marmosets (Callithrix jacchus). J Neural Transm 107:799-814. https://doi.org/10. 1007/s007020070060

24. McMillan P, Korvatska E, Poorkaj P, Evstafjeva Z, Robinson L, Greenup L, Leverenz J, Schellenberg GD, D'Souza I (2008) Tau isoform regulation is region- and cell-specific in mouse brain. J Comp Neurol 511:788-803. https://doi.org/10.1002/cne.21867

25. Mestre-Francés N, Serratrice N, Gennetier A, Devau G, Cobo S, Trouche SG, Fontès P, Zussy C, De Deurwaerdere P, Salinas S, Mennechet FJD, Dusonchet J, Schneider BL, Saggio I, Kalatzis V, Luquin-Piudo MR, Verdier J-M, Kremer EJ (2018) Exogenous LRRK2G2019S induces parkinsonian-like pathology in a nonhuman primate. JCI Insight 3:e98202. https://doi.org/ 10.1172/jci.insight.98202

26. Meyer-Luehmann M, Coomaraswamy J, Bolmont T, Kaeser S, Schaefer C, Kilger E, Neuenschwander A, Abramowski D, Frey P, Jaton AL, Vigouret JM, Paganetti P, Walsh DM, Mathews PM, Ghiso J, Staufenbiel M, Walker LC, Jucker M (2006) Exogenous induction of cerebral beta-amyloidogenesis is governed by agent and host. Science 313:1781-1784. https://doi.org/ 10.1126/science.1131864

27. Nadkarni NA, Bougacha S, Garin C, Dhenain M, Picq JL (2019) A 3D population-based brain atlas of the mouse lemur primate with examples of applications in aging studies and comparative anatomy. Neuroimage 185:85-95. https://doi.org/10.1016/j.neuroimage.2018.10.010

28. Nelson PT, Alafuzoff I, Bigio EH, Bouras C, Braak H, Cairns NJ, Castellani RJ, Crain BJ, Davies P, Del Tredici K, Duyckaerts C, Frosch MP, Haroutunian $\checkmark$, Hof PR, Hulette CM, Hyman BT, Iwatsubo T, Jellinger KA, Jicha GA, Kovari E, Kukull WA, Leverenz JB, Love S, Mackenzie IR, Mann DM, Masliah E, McKee AC, Montine TJ, Morris JC, Schneider JA, Sonnen JA, Thal DR, Trojanowski JQ, Troncoso JC, Wisniewski T, Woltjer RL, Beach TG (2012) Correlation of Alzheimer disease neuropathologic changes with cognitive status: a review of the literature. J Neuropath Exp Neur 71:362-381. https://doi.org/10.1097/NEN.0b013e31825018f7

29. Parvizi J, Van Hoesen GW, Buckwalter J, Damasio A (2006) Neural connections of the posteromedial cortex in the macaque. Proc Natl Acad Sci USA 103:1563-1568. https://doi.org/10.1073/pnas.0507729103

30. Pearson JM, Heilbronner SR, Barack DL, Hayden BY, Platt ML (2011) Posterior cingulate cortex: adapting behavior to a changing world. Trends Cogn Sci 15:143-151. https://doi.org/10.1016/j.tics.2011.02.002

31. Picq JL, Villain N, Gary C, Pifferi F, Dhenain M (2015) Jumping stand apparatus reveals rapidly specific age-related cognitive impairments in mouse lemur primates. PLOS ONE 10:e0146238. https://doi.org/10.1371/journal. pone.0146238

32. Purro SA, Farrow MA, Linehan J, Nazari T, Thomas DX, Chen ZC, Mengel D, Saito T, Saido T, Rudge P, Brandner S, Walsh DM, Collinge J (2018) Transmission of amyloid-beta protein pathology from cadaveric 
pituitary growth hormone. Nature 564:415. https://doi.org/10.1038/ s41586-018-0790-y

33. Ragozzino ME, Ragozzino KE, Mizumori SJY, Kesner RP (2002) Role of the dorsomedial striatum in behavioral flexibility for response and visual cue discrimination learning. Behav Neurosci 116:105-115. https://doi.org/10 1037/0735-7044.1 16.1.105

34. Ridley RM, Baker HF, Windle CP, Cummings RM (2006) Very long term studies of the seeding of beta-amyloidosis in primates. J Neural Transm 113:1243-1251. https://doi.org/10.1007/s00702-005-0385-2

35. Rolls ET (2000) The orbitofrontal cortex and reward. Cereb Cortex 10:284-294. https://doi.org/10.1093/cercor/10.3.284

36. Sawiak SJ, Picq JL, Dhenain M (2014) Voxel-based morphometry analyses of in vivo MRI in the aging mouse lemur primate. Front Aging Neurosci 6:82. https://doi.org/10.3389/fnagi.2014.00082

37. Silhol S, Calenda A, Jallageas V, MestreFrances N, Bellis M, Bons N (1996) B-Amyloid protein precursor in Microcebus murinus: genotyping and brain localization. Neurobiol Dis 3:169-182. https://doi.org/10.1006/nbdi. 1996.0017

38. Simic G, Leko MB, Wray S, Harrington C, Delalle I, Jovanov-Milosevic N, Bazadona D, Buee L, de Silva R, Di Giovanni G, Wischik C, Hof PR (2016) Tau protein hyperphosphorylation and aggregation in Alzheimer's disease and other tauopathies, and possible neuroprotective strategies. Biomolecules. https://doi.org/10.3390/biom6010006

39. Vogt BA, Pandya DN (1987) Cingulate cortex of the Rhesus-monkey.2. Cortical afferents J Comp Neurol 262:271-289. https://doi.org/10.1002/ cne. 902620208

\section{Publisher's Note}

Springer Nature remains neutral with regard to jurisdictional claims in published maps and institutional affiliations.
Ready to submit your research? Choose BMC and benefit from:

- fast, convenient online submission

- thorough peer review by experienced researchers in your field

- rapid publication on acceptance

- support for research data, including large and complex data types

- gold Open Access which fosters wider collaboration and increased citations

- maximum visibility for your research: over $100 \mathrm{M}$ website views per year

At BMC, research is always in progress.

Learn more biomedcentral.com/submissions 